ACCEPTED MANUSCRIPT

\title{
Flow Control of Non-Slender Delta Wings by Leading-Edge Modifications
}

To cite this article before publication: Martin Rein et al 2020 Fluid Dyn. Res. in press https://doi.org/10.1088/1873-7005/ab67d8

\section{Manuscript version: Accepted Manuscript}

Accepted Manuscript is "the version of the article accepted for publication including all changes made as a result of the peer review process, and which may also include the addition to the article by IOP Publishing of a header, an article ID, a cover sheet and/or an 'Accepted Manuscript' watermark, but excluding any other editing, typesetting or other changes made by IOP Publishing and/or its licensors"

This Accepted Manuscript is $(2020$ The Japan Society of Fluid Mechanics and IOP Publishing Ltd

During the embargo period (the 12 month period from the publication of the Version of Record of this article), the Accepted Manuscript is fully protected by copyright and cannot be reused or reposted elsewhere.

As the Version of Record of this article is going to be / has been published on a subscription basis, this Accepted Manuscript is available for reuse under a CC BY-NC-ND 3.0 licence after the 12 month embargo period.

After the embargo period, everyone is permitted to use copy and redistribute this article for non-commercial purposes only, provided that they adhere to all the terms of the licence https://creativecommons.org/licences/by-nc-nd/3.0

Although reasonable endeavours have been taken to obtain all necessary permissions from third parties to include their copyrighted content within this article, their full citation and copyright line may not be present in this Accepted Manuscript version. Before using any content from this article, please refer to the Version of Record on IOPscience once published for full citation and copyright details, as permissions will likely be required. All third party content is fully copyright protected, unless specifically stated otherwise in the figure caption in the Version of Record.

View the article online for updates and enhancements. 


\title{
Flow Control of Non-Slender Delta Wings by Leading-Edge Modifications
}

\author{
Martin Rein ${ }^{1}$, Felix Busse, Frank Edzards, Hartmut Haselmeyer, \\ Gebhard Höhler and Lars Siegel \\ DLR German Aerospace Center, Institute of Aerodynamics and Flow Technology \\ Bunsenstr. 10, 37073 Göttingen, Germany
}

\begin{abstract}
Different concepts for controlling a non-slender delta wing are considered and compared with each other. The concepts are based on geometrical modifications of the leading edges. The strategy aims at producing an asymmetric distribution of lift due to vortices on the left and right wing half and thus, for example, rolling moments to be used in controlling the model. The effectiveness of different approaches is determined in low speed wind tunnel tests both by measuring forces and moments acting on the model and by investigating the flow field around the model by particle image velocimetry and oilflow visualization of skin friction lines. Two approaches are pursued. One is based on static and dynamic actuators acting at the left leading edge. The other uses different combinations of leading edge shapes on the two halves of the model. Finally, the effect of asymmetric boundary layer tripping is briefly considered.
\end{abstract}

Keywords: delta wing, flow control, stability and control, vortical flow, vortex, leading edge, separation

\section{Introduction}

The aerodynamics of delta wings is dominated by large-scale vortices that can form on the lee side due to flow separation at the leading edges. The vortices provide additional lift, the so-called vortex lift. Flows around rounded leading edges result in leading-edge suction that contributes to a reduction in drag. This opens the possibility of utilizing novel concepts for the control and stability of delta wings by deliberately manipulating the vortical flow at the leading edge. Such approaches can be particularly useful at greater angles of attack when the effect of classical control surfaces at the trailing edge becomes ineffective because the control surfaces are immersed in a regime of separated flow.

The formation of the vortices above delta wings depends on the leading-edge sweep angle. Shear layers that separate from the leading edge form primary vortices and initially reattach to the lee side of the wing outboard of the symmetry plane. With increasing angle of attack the primary reattachment lines move towards the symmetry plane. If the sweep angle is small the symmetry plane is never reached. This is characteristic of nonslender wings. For greater sweep there exists an angle of attack above that a direct reattachment to the surface of the wing no longer occurs. The two shear layers meet at a saddle point and, thereafter, reattach at the centreline of the wing. Primary vortices of non-slender wings are located closer to the wing than those of slender wings. Non-slender wings have a sweep angle of less than about $55^{\circ}$ (Gursul et al., 2007).

${ }^{1}$ Corresponding author, email: martin.rein@dlr.de 
At sharp leading edges the location of separation is fixed and shear layer separation occurs along the whole leading edge. This no longer holds for rounded geometries where, with increasing angle of attack, flow separation begins near the trailing edge and then the onset of separation progresses upstream towards the apex of the delta wing (Luckring, 2010). On both wing halves, the separating shear layers roll up forming two longitudinal primary vortices of opposite circulation with high axial velocities and correspondingly low pressures in the vortex cores. Vortices formed at sharp leading edges are stronger and located slightly more inboard than those originating from blunt edges (Luckring, 2010, Verhaagen, 2012, Luckring and Hummel, 2013).

Further downstream primary vortices can burst. The radial extent of the vortices increases and the axial velocity decreases strongly. This is connected with a reduction in vortex lift. At small angles of attack vortex breakdown first occurs near the trailing edge. With increasing angle of attack the location of vortex breakdown moves upstream, eventually reaches the apex and the wing stalls (Wentz and Kohlman, 1971). With sharp leading edges the onset of stall occurs at a smaller angle of attack than with rounded ones (Verhaagen, 2012).

On the lee-side surface of the wing the spanwise flow of the primary vortices encounters a positive pressure gradient. This causes a secondary separation and the formation of a secondary vortex near the leading edge. Its circulation is opposite to that of the primary vortex. For the formation of secondary vortices the state of the boundary layer, laminar or turbulent, is important (Hummel, 2004, Furman and Breitsamter, 2013).

Longitudinal vortices not only occur on swept wings, but are also formed at wingtips or behind obstacles, and are used in swirl injectors. Hence, a control of longitudinal vortices is of interest in a variety of different applications. A general survey of control techniques has been given by Bushnell (1992). He distinguishes between controlling pressure gradients that cause vortices, segmenting the production of vortices, exciting instability modes, extracting energy from vortices and using other vortices for control. The effect of vortices on the aerodynamics of delta wings is governed by the strength and position of the vortices, and particularly by the location of vortex breakdown that is connected with a reduction in lift. Control techniques of vortex breakdown on delta wings have been reviewed by Mitchell and Delery (2001) and control mechanisms of leading edge vortices by Gursul et al. (2007). In general, these techniques can be divided into pneumatic, mechanical, and energetic approaches. Furthermore, stationary and unsteady or periodic methods need to be distinguished.

A well-known pneumatic approach for delaying vortex breakdown on delta wings is along-the-core blowing (Gursul et al., 2007). Lift can be augmented and stall delayed by oscillatory blowing at the leading edge (Williams et al., 2008). Renac et al. (2005) showed that one sided oscillatory blowing at a rounded leading edge 
can produce significant rolling moments. For appropriately chosen frequencies Kölzsch and Breitsamter (2014) found that pulsed blowing leads to a stabilization of the leading edge vortex and a shift of vortex breakdown in downstream direction. Erickson (2017) applied flow-through porosity to leading edges and showed that this passive approach weakened the wing vortices. Finally, also classical approaches of lift augmentation, for example, circulation control by blowing, have been tested successfully on delta and diamond wings and demonstrated a potential for roll control (Frith and Wood, 2006).

Other unsteady methods use small oscillating flaps at the leading edge. Deng and Gursul (1997) observed a hysteretic dependence on the amplitude of the location of vortex breakdown. Kölzsch (2017) applied two groups of three miniature flaps on one wing half thus producing a rolling moment. The most significant effect, however, was observed in the post stall region where the excitation caused an increase in both lift and drag with unsteady excitation being more effective than a stationary deflection.

Stationary leading-edge flaps have been used to reduce drag by generating suction at the leading edge (vortex flaps, Rao (1979)) and to enhance lift by controlling strength and position of the vortices (Karagounis et al., 1989). In the former case, suction is concentrated on the upper surface of a downward deflected flap. In the latter case, the vortex sheet is shed from an upward deflected flap. Other leading edge devices like fences, pylons and slots have been explored by Rao and Johnson (1981). The structure of the vortices can also be changed by manipulating the separating shear layer directly at the leading edge. In wind tunnel tests, Matsuno et al. (2002) positioned a rigid micro-flap on one half of a non-slender delta wing. They found a non-linear dependence of the rolling moment on the azimuthal position of the flap along the circular leading edge. The corresponding flow fields have subsequently been analysed numerically by Kaiden and Nakamura (2004). Lee et al. (2000) applied microelectromechanical systems (MEMS) actuators and also micro-flaps to a circular leading edge and showed that asymmetric arrangements can generate appreciable rolling moments. In order to apply the MEMS actuators at appropriate locations Huang et al. (2001) sensed the course of the separation line by shear stress sensors prior to actuation.

As already noted above, location and strength of delta wing vortices depend on the shape of the leading edge. However, not only the difference between sharp and rounded shapes but also the way a sharp edge is bevelled or the radius of curvature of rounded edges is important. This has been studied, for example, by Miau et al. (1995), Lowson and Riley (1995), Verhagen (2012) and in the Second International Vortex Flow Experiment (VFE2, Luckring and Hummel (2013)). Changes in leading edge shapes can be realised by morphing (Vasista et al., 2017). The potential of more general morphing concepts for control has been shown 
for a generic lambda wing configuration (SACCON) by Paul and Rein (2017). The leading edge shape of the SACCON configuration varies between sharp and rounded segments. For a relatively thick symmetric diamond wing Hövelmann and Breitsamter (2015) studied the effect of spanwise varying leading edge shapes. Vortex formation was observed only at segments with sharp leading edges. Intermediary rounded edges caused a segmentation of vortices. A segmentation of vortices can also be realized by sinusoidal leading edges. According to Chen and Wang (2014) stall can be delayed in this manner. The related complex flow topology has been analysed by Goruney and Rockwell (2009).

Another concept of flow control is using moving surfaces. The associated addition of momentum can significantly delay separation (Modi, 1997). Azuma and Nakamura (2002) explored this approach numerically and found that a rotating cylindrical leading edge can increase lift of a delta wing in the stall regime. Momentum injection at the leading of delta wings has also been accomplished by dielectric barrier discharge actuators (DBD actuators). Wilke (2009) applied a DBD actuator at a rounded leading edge using the same thin model as in the present paper. With increasing angle of attack he observed first an increase and later a reduction of lift. PIV measurements by Greenblatt et al. (2008) showed a reformation of yortices in the deep stall regime of a delta wing with sharp leading edges if a DBD actuator was periodically excited.

In the present paper, different concepts for controlling a delta wing are reviewed that have been tested in the course of the last years at the Institute of Aerodynamics and Fluid Mechanics of DLR. The concepts are based on geometrical modifications of the leading edge. The goal is to change strength and position of vortices by slight modifications of the wings' leading edges. The strategy aims at producing an asymmetric distribution of lift on the left and right wing half and thus, for example, rolling moments to be used in controlling the model. The effectiveness of different approaches is determined in low-speed wind tunnel tests both by measuring forces and moments acting on the model and by investigating the flow field around the model by particle image velocimetry and oil-flow visualization of skin friction lines. The focus is on the lateral force and moments but also longitudinal coefficients will be discussed to some extent. Two approaches are pursued. One is based on static and dynamic actuators acting at the left leading edge. The other uses different combinations of leading edge shapes on the two halves of the model. Finally, the effect of boundary layer tripping is briefly considered. In the following sections, first, the two wind tunnel models and the experimental setup are described. Then, experimental results are presented and analyzed. This is followed by a conclusion. 


\section{Experimental setups and procedures}

\subsection{Delta wing models}

Wind tunnel tests have been performed with two non-slender flat-plate delta wing models of same planform but different thickness. The leading edges of the thinner model, called the "thin model" hereafter, can be exchanged. The thicker "actuator model" provides space inside the model for a motor that can be used for actuating the leading edge of the left wing half. Fig. 1 shows the shared geometrical dimensions of the two wing planforms. The sweep angle of both models is $A=53^{\circ}$ and their wing half span equals $s=250 \mathrm{~mm}$. Thus, the root chord is $c=331.8 \mathrm{~mm}$, the mean aerodynamic chord $l_{\mu}=221.2 \mathrm{~mm}$, the neutral point $x_{N 25}=\mathrm{c} / 2=166 \mathrm{~mm}$ and the planform area $S_{r e f}=82940 \mathrm{~mm}^{2}$. Upper and lower surfaces of the models are parallel planes, i.e., the wings are not cambered. Trailing edges of both models are beveled, at an angle of $20^{\circ}$ (thin model) and $40^{\circ}$ (actuator model), with the slant on the lower side. The thickness of the thin model is $t=10 \mathrm{~mm}$ and that of the actuator model $t=20 \mathrm{~mm}$. This corresponds with a thickness to chord ratio of $t / c=0.03$ for the thin model and $t / c=0.06$ for the actuator model. In both cases a balance can be fixed to the model via a seat attached to the lower (windward) side of the model. The model is held by a rear sting that is either attached to the balance or to a dummy replacing the balance. Both models are made of steel.

The thin model can be equipped with leading edges of various geometries. Initial tests were performed with circular and elliptic shapes. The radius of the circular leading edge equals to one half of the thickness of the model. The elliptic leading edge is formed by an ellipse whose semi-minor axis also equals to one half of the thickness of the model, and its aspect ratio is $2: 1$. Hence, the radius of curvature of the elliptic leading edge is half the size of that of the circular one, i.e., it equals to a quarter of the wing thickness. At the transition to the plane upper and lower surfaces there is an abrupt transition from a circular and elliptic shape, respectively, to a planar surface, i.e., both leading edge shapes have are discontinuity in curvature at this location.

In succeeding tests of the thin model, leading edges whose shape can vary between round and sharp regions along either half of the model (left and right) have been used (see Fig. 2a). Special care was taken to provide for a smooth transition to the plane upper and lower surface of the model (no discontinuity in curvature). Apart from the smooth transition, the round geometry resembles the elliptic shape described above. If the leading edge is sharp, the sharp edge is located at the height of the upper surface. On the lower side a slant of initially $47^{\circ}$ merges smoothly into a curved transition to the lower surface. On either half of the model the shape of the leading edge can vary between sharp and round. Sections with a sharp leading edge will be denoted by "S" and those with round ones by "R" hereafter. The transition between sharp and round happens either abruptly or gradually using a ramp function (for an intermediate shape see Fig. 2a). The transition occurs in the middle of 
the leading edge, in the case of gradual transitions over about $20 \%$ of the length of the leading edge. In summary, four different symmetric leading edge shapes are available for each wing half: fully sharp (SSSS), sharp at the apex and round at the wing tip (RSSR), round at the apex and sharp at the wing tip (SRRS), and fully round (RRRR). These are shown in Fig. $2 b$ for configurations with gradual transitions in leading edge geometry. In addition, asymmetric configurations as, for example, RSSS or SRRR are considered. If the transition between a sharp and round leading edge contour happens abruptly this is denoted by a bar: S|RRR or R|SSS. The effect of abrupt transitions is only studied with differently shaped left leading edges, the right leading edge is either fully sharp or fully round.

The actuator model is equipped with round leading edges of circular shape. With the thickness of the actuator model being twice as large as that of the plane model, also the radius of curvature is twice as large as that of the corresponding thin model. The left leading edge of the actuator model consists of a module that can be exchanged. Basically, two different modules have been designed and built, one with a protruding bar (Fig. 3a) and another one with a rotatable cylinder (Fig. 3b). Both, bar and cylinder, extend along $58 \%$ of the leading edge, starting at a position as close to the apex of the model as the design allows (bar: $1.6 \%$ or $x / c=0.02$, cylinder: $5 \%$ or $x / c=0.06$ ). Near the wing tip the remaining space is used for the driving parts.

A protruding bar provides a well-defined location of flow separation when the model is at a non-zero angle of attack. In its most inboard position the outer surface of the bar follows the circular shape of the leading edge. The bar's maximum protrusion into the flow is $0.73 \mathrm{~mm}$ and its thickness $1.5 \mathrm{~mm}$. The bar can oscillate back and forth at frequencies up to $150 \mathrm{~Hz}$, thus, providing an unsteady forcing of the separating shear layer.

The module with a rotatable cylinder replaces a part of the left leading edge with a cylinder of the same radius as that of the baseline. Two different cylinders have been considered, a rough and a smooth one. The rough cylinder can be rotated at a rate of up to $99 \mathrm{~Hz}$ in both directions. This corresponds with a surface velocity of $\pm 6.22 \mathrm{~m} / \mathrm{s}$, thus accelerating or decelerating the boundary layer. However, the rough cylinder has not yet been tested. The smooth cylinder is only used stationary. It has a notch of $1 \mathrm{~mm}$ depth milled into its surface along its length (parallel to its axis, see Fig. 3b). The upper edge of the notch is sharp and at its lower side the cylinder surface transitions smoothly from the notch towards the cylinder radius. As with the protruding bar, the sharp edge is intended to affect flow separation at a defined location when the model is at an angle of attack. By turning the cylinder the location of separation is changed. The angular position of the edge is measured by the setting angle $\varphi$, with $\varphi=0^{\circ}$ when the edge is at the apex of the leading edge. $\varphi$ increases in clockwise direction 
(see Fig. 3b). At $\varphi=180^{\circ}$ the notch disappears completely inside the model. Therefore, this case is taken to correspond to the baseline configuration.

In addition to changing the leading edge geometry, leading edge fences have been attached to the thin model with circular leading edge in order to obtain a benchmark for effects caused by changing the geometry of the leading edge. The fences were aligned parallel to the leading edge of the left wing half, right at the transition between the round edge and the plane surface. Details on their size and location are reported below together with their effect.

In certain cases the boundary layer has been tripped at the leading edge (Edzards, 2014). A row of trip dots was used. The height of the dots was $183 \mu \mathrm{m}$ and both their diameter and the spacing between them was about $1.25 \mathrm{~mm}$. The exact locations used for tripping are specified in the discussion of the corresponding results.

\subsection{Measurement techniques}

Integral effects of leading edge modifications are assessed by comparing associated aerodynamic coefficients with those corresponding with baseline configurations. To this end, forces and moments are determined using a six component strain gauge. Mean values of forces and moments are obtained by averaging the signals of the balance over an interval of $10 \mathrm{~s}$. A cylindrical 0.75 " Task balance is mounted via a seat underneath the model. Maximum deviations of forces and moments from the target values as determined during the calibration of the balance translate to non-dimensional margins of error that depend on freestream conditions. Under the tested conditions maximum error margins of non-dimensional coefficients of forces and moments are approximately given by $\Delta c_{x}=2 \times 10^{-3}-2 \cdot 10^{-2}, \Delta c_{y}=2 \times 10^{-2}-2 \times 10^{-1}, \Delta c_{z}=2 \times 10^{-2}-2 \times 10^{-1}, \Delta c_{l}=$ $6 \times 10^{-4}-7 \times 10^{-3}, \Delta c_{m}=2 \times 10^{-3}-2 \times 10^{-2}$, and $\Delta c_{n}=10^{-3}-10^{-2}$, the smaller values corresponding with $u_{\infty}=50 \mathrm{~m} / \mathrm{s}$ and the larger with $u_{\infty}=15 \mathrm{~m} / \mathrm{s}$ where $u_{\infty}$ is the freestream velocity.

Forces are scaled by the product of the freestream dynamic pressure $q_{\infty}$ and the planform area $S_{\text {ref. }}$ The rolling and yawing moment are scaled with the wing half span $s$ while the pitching moment is scaled using the mean aerodynamic chord $l_{\mu}$. The neutral point $x_{N 25}$ is chosen as moment reference point. Force and moment coefficients are presented in the model fixed coordinate system. In addition, lift and drag coefficients will be cited. The Reynolds number is formed with the mean aerodynamic chord, $\operatorname{Re}=\rho_{\infty} u_{\infty} l_{\mu} / \mu$, where $\rho_{\infty}$ and $\mu$ are the freestream density and the dynamic viscosity.

In some tests, main topological structures in the velocity fields were investigated using stereoscopic particle image velocimetry (SPIV). All three components of the mean velocity were determined in selected planes above the delta wing. The planes were orientated perpendicularly to the freestream velocity vector. Hence, at non-zero 
angle of attack the planes are not perpendicular to the model surface. Their position is specified in the reference frame of the model. The corresponding longitudinal position $x$ is scaled by the root chord $c$ with $x / \mathrm{c}=0$ denoting the apex of the model and $x$ increasing in downstream direction. Where applicable, the spanwise coordinate $y$ is scaled by the local wing half span $s(x)$.

A laser-light sheet has been formed using an Nd:Yag-laser (wavelength $\lambda=532 \mathrm{~nm}$ ). Both, laser light-sheet optics and cameras were mounted on a movable traverse above the test section. In this manner the position of the measurement plane could easily be changed. The laser beam of the stationary laser was coupled to the movable light-sheet optics by a system of mirrors. At the model surface the sheet had a thickness of $\Delta x \approx 3 \mathrm{~mm}$. The two cameras have been arranged downstream of the model, outside of the test section, at a vertical position $50 \mathrm{~mm}$ above the model's surface at zero angle of attack. Scheimpflug adapters were angled at about $80^{\circ}$. Overall, this resulted in a resolution of $4.8 \mathrm{pixel} / \mathrm{mm}$. Typically 50 double images were taken, evaluated and subsequently averaged. Initial tests showed no qualitative differences in the results when a greater number of 100 or 200 double images was used. All images were evaluated using the program DaVis 8.1 of LaVision with $32 \times 32$ pixel interrogation windows, and an overlap of $50 \%$ (actuator model) and $70 \%$ (thin model), respectively. The final error in the velocity magnitude is estimated to be less than $5 \%$. Disturbances by light reflected off the model surface have been reduced (but not fully avoided) by covering the models by a matt black finish in all PIV tests. The flow was seeded with oil droplets of approximately $1 \mu \mathrm{m}$ diameter in the diffuser of the closed circuit wind tunnel. For details see Siegel (2013) and Busse (2013).

The position and extent of vortices has also been qualitatively visualized using surface flow visualization by the fluorescent-oil film method. In this manner an overview of skin friction lines on the lee side of the model is obtained. From the skin friction lines, separation and reattachment lines are identified thus providing footprints of vortices.

\subsection{Low speed wind tunnel}

All experiments have been performed in the low-speed wind tunnel $1 \mathrm{mG}$ of the DLR Institute of Aerodynamics and Flow Technology in Göttingen. The $1 \mathrm{mG}$ is a closed circuit (Göttingen type) atmospheric tunnel. A rectangular nozzle $(1.05 \mathrm{~m} \times 0.70 \mathrm{~m})$ was used and the length of the open test section was $1.35 \mathrm{~m}$. The contraction ratio of the nozzle is $1: 4.8$. A maximum velocity of $60 \mathrm{~m} / \mathrm{s}$ can be achieved. This relates to Reynolds numbers formed with ten percent of the square root of the cross sectional area $A$ of the test section, $\operatorname{Re}_{0.1} v_{(A)}$, of $\operatorname{Re}_{0.1}(A) \leq 0.35 \times 10^{6}$. In the present tests the velocity was varied in the range $u_{\infty}=15-55 \mathrm{~m} / \mathrm{s}$ corresponding with Reynolds numbers $\operatorname{Re}=2.2-7.9 \times 10^{5}$ (here, $\operatorname{Re}$ is based on the mean aerodynamic chord). The turbulence 
level of the tunnel is less than 0.0015 . The model was mounted on a rear sting that was fixed to a support allowing for changing the angle of attack.

\section{Symmetric models and benchmark tests}

\subsection{Symmetric configurations}

Symmetric delta wings with same leading edge shapes on both, left and right wing half, are considered as baseline configurations hereafter. Different shapes of the leading edge are considered that result in slightly different longitudinal forces and moments. This is shown for lift, drag and pitching moment coefficients of the thin model with circular, elliptic and sharp leading edge in Fig. 4a. Note that the cowling of the balance underneath the model produces an effective camber that causes the lift to become negative at small angles of attack. Differences in the respective lift and drag coefficients are mainly caused by the axial force (model fixed system). With increasing angle of attack the flow around the various round leading edges induces a suction force that is directed forward. This force depends both on the radius of curvature and the area affected. For the sharp leading edge the mechanism of suction does not work and, hence, the corresponding drag is greater than in the case of round leading edges. Similarly, with sharp leading edges the wing stalls earlier than with round leading edges (Verhaagen, 2012).

At zero sideslip and roll angle, the lateral force and moments should disappear. Within the error bounds specified above this is satisfied for the yawing moment and the side force (at least as long as the wing is not fully stalled). The rolling moment of the thin model, however, shows some peculiar behavior, both for the circular and the elliptic leading edge. Already at certain small values of the angle of attack, $\alpha$, the rolling moment becomes non-zero within narrow $\alpha$-intervals (see Fig. 4b). Both, peaks in positive and negative direction occur. The location of the peaks has been reproduced in different wind tunnel entries and is therefore believed not to be caused by a possible slight misalignment of the model. At the last peak, at about $\alpha=7^{\circ}$, a hysteretic behaviour has been observed - the peak occurs only when the angle of attack is increased, but not when it is decreased. In order to discover whether peaks are caused by minute discrepancies in the leading edge geometry a test has also been performed with both leading edges being taped by a smooth thin film. This reduced the height of the spikes (cf. Fig. 4b) but did not fully remove the peaks. Further, the direction of the peak at $\alpha \approx 7^{\circ}$ is reversed to positive values. The exact location of peaks with respect to angle of attack depends on the velocity, i.e., Reynolds number (see Fig. 4c). With increasing velocity their first occurrence moves to greater angles of attack. It is conjectured that the peaks are at least partly caused by an asymmetric onset of flow separation and vortex formation at the left and right leading edge, with the resulting imbalanced vortex lift 
resulting in a rolling moment. Further, for non-slender wings that are considered in the present case, vortex breakdown will pass the trailing edge of the model already at small angles of attack (Wentz and Kohlman, 1971). Again, it is likely that this will not occur in a fully symmetric manner thus resulting in an asymmetric distribution of vortex lift as soon as vortex breakdown occurs on one wing half.

As will be seen below, measures of leading edge flow control considered in this study typically take effect at angles of attack greater than those where peaks are observed. Therefore, an interference of the phenomenon of peak formation with measures of flow control is not expected.

\subsection{Benchmark: leading edge fences}

In order to obtain a quantitative estimate of changes in aerodynamic coefficients that are achievable with more conventional leading edge devices, the effect of leading edge fences placed on the top surface of the wing has been tested and determined. Rolling moments and also other lateral effects are generated when a leading edge fence is applied on one wing half only. Fences of different size have been tested at different locations to provide a benchmark against which other control approaches can be measured. Width and height of the largest fence was $100 \mathrm{~mm} \times 10 \mathrm{~mm}$, and another two were sized $50 \mathrm{~mm} \times 10 \mathrm{~mm}$ and $50 \mathrm{~mm} \times 5 \mathrm{~mm}$. These widths correspond with $24 \%$ and $12 \%$ of the length of the leading edge. All fences were placed on the left half of the thin wing with circular leading edge, $5 \mathrm{~mm}$ behind the apex of the round edge, i.e., right at the transition between curved and flat surface. The outer edge of the wide fence was placed at the wing tip and in the case of the narrower ones either also at the wing tip or $50 \mathrm{~mm}$ inwards. The fences were made from 90 -degree angle plates that were glued to the wing.

Rolling and yawing moments generated by the different leading edge fences are compared with each other and that of the clean wing (baseline) in Fig. 5. The rolling moment is mainly increased at intermediate angles of attack while the effect on the yawing moment is greatest when the model is at no incidence and then decreases strongly with increasing angle of attack. In both cases maximum changes are of the order of 0.01. Axial and side forces are also appreciably changed, the pitching moment, however, is little affected. Not surprisingly, the effect of the fences correlates with their size. It may seem less plausible that the outer placement of the small fence (fence 4) causes a smaller rolling moment than an inner placement (fence 3). A new vortex is formed at the outer edge of a fence (Paul and Rein, 2017). With an inner placement of the fence the new vortex still lies above the wing resulting in vortex lift and thus potentially in an enhancement of the rolling moment. The results of these considerations suggest that producing non-dimensional rolling and yawing moments of the order of 0.01 can be considered as a target value for other measures of flow control. 


\section{Actuator model}

\subsection{Leading edge with protruding bar}

The protruding bar extends over the greater part of the left leading edge and can be used either stationary or in an oscillating mode. The concept of the bar is to introduce an asymmetry into the configuration that results in a non-zero lateral force and lateral moments that can be used for controlling the wing. The stationary bar provides a well-defined location of flow separation when the bar is deployed and an unchanged round leading edge when it is retracted. With asymmetric flow separation on the two halves of the delta wing the pair of vortices formed by the separating shear layers is no longer symmetric. Different strengths and positions of the two vortices can thus result in non-zero lateral forces and moments. In unsteady applications oscillation frequencies of the bar are of the same order as that of the instability of separating shear layers (Menke et al., 1999). A one-sided forcing of the shear layer can affect the breakdown behavior of the respective vortex and thus change the magnitude of vortex lift on the corresponding side, again resulting in non-zero lateral coefficients. These measures also have an impact on longitudinal coefficients. Lift increases and drag and pitching moment decrease (see Fig. 6). The effect may be related to that of leading-edge Gurney flaps on delta wings (Traub and Galls, 1999).

In Fig. 7a the rolling moment coefficient resulting from a protruding bar is compared with that of the clean configuration (bar retracted) for a freestream velocity of $u_{\infty}=30 \mathrm{~m} / \mathrm{s}$. Results of three angle of attack runs each are plotted in order to show repeatability. Within certain ranges of angle of attack the bar as a control mechanism produces a rolling moment of sufficient magnitude, however, in between there is a gap where it does not - actually the rolling moment even changes sign. The behavior is pretty much the same at lower and higher velocities.

The effect of an oscillating bar is depicted in Fig. $7 \mathrm{~b}$. The freestream velocity is again $u_{\infty}=30 \mathrm{~m} / \mathrm{s}$ and frequencies range from $f=10-150 \mathrm{~Hz}$, corresponding with Strouhal numbers $\mathrm{St}=f c / u_{\infty}=0.33-1.66$. For comparison, results are also shown for the baseline (clean) configuration and a leading edge with a stationary protruded bar $(\mathrm{St}=0)$. With oscillating bar maximum values of the rolling moment are similar to or smaller than that caused by the stationary bar. At the first maximum, at $\alpha \approx 13^{\circ}-14^{\circ}$, the rolling moment initially increases with frequency but for $\mathrm{St}>1.11$ it begins to decrease again. This occurrence of a maximum value of the rolling moment at $\mathrm{St} \approx 1$ together with particularly large values at high angle of attacks suggests that the mechanism of unsteady forcing does work. The outcome at different velocities but same non-dimensional frequency is shown in Fig. $7 \mathrm{c}$ for $\mathrm{St}=0.66$. With increasing velocity the effect of the oscillations becomes greater but in a slightly irregular manner. However, a superposition of Reynolds number effects cannot be excluded. 
In order to obtain some insight into the origin of rolling moments, oil-flow pictures have been produced for a velocity of $u_{\infty}=30 \mathrm{~m} / \mathrm{s}$ and selected angles of attack. An example is reproduced in Fig. 8. Reattachment lines and secondary separation lines characteristic of delta wing flows are visible. Note, that when a bar is deployed an additional structure appears at the leading edge downstream of the bar, thereafter propagating inward. This could indicate a region of separated flow or a new vortex at the left wing tip. A qualitative visualization by smoke has supported the interpretation as a new vortex. Similar features are also observed with the notched rotatable cylinder and will be discussed more deeply in the next section were also PIV results are provided.

From the above, it becomes clear that a protruding bar is not useful for controlling a delta wing. The nonuniform dependence of the effect of the protruding bar on the angle of attack is due to a fixed location of flow separation. A more flexible approach is introduced in the next section.

\subsection{Leading edge with rotatable cylinder}

The basic concept of the cylinder with notch is similar to that of the protruding bar, i.e., affecting flow separation. The additional freedom of rotating the cylinder about its axis presents the advantage that the location of the disturbance can be adjusted according to the current angle of attack and possibly other parameter such as flow velocity or angle of yaw.

The effect of the angular position of the notch on the rolling and pitching moment is illustrated in Fig. 9a/b, again for $u_{\infty}=30 \mathrm{~m} / \mathrm{s}$. At this velocity the notch is most effective for $\varphi=45^{\circ}$ and to a lesser extent for $\varphi=0^{\circ}$. Maximum values of the rolling moment are greater than the largest ones obtained with the protruding bar. At the other tested orientations the effect is nil. A gap in the rolling moment, as occurring with the protruding bar at certain angles of attack, is no longer observed. A positive rolling moment means an increase of lift on the left as compared to the right wing half. Since the increase of the rolling moment is accompanied by a decrease of the pitching moment (see Fig. 9b) the contribution to lift of the rear part of the wing becomes more important when the actuator is effective.

When the notch lies on the windward side of the model, below the stagnation line, it has little or no effect on boundary layer separation with succeeding vortex formation. Similarly, when the notch lies on the lee side at a position downstream of the separation line, its effect on the separating shear layer is reduced. This explains the ineffectiveness of the notch at certain angular positions. However, not only the orientation of the notch but also the freestream velocity (Reynolds number) plays a role. This is shown in Fig. 9c for the example of the notch at a fixed angle of $\varphi=90^{\circ}$. Only when the freestream velocity exceeds a limiting value the rolling moment becomes affected. Here, with increasing Reynolds number separation is delayed. The separation line moves in 
the direction of increasing $\varphi$, approaching and finally interacting with the notch. The most effective orientation of the cylinder thus depends on both the angle of attack and the velocity.

Rolling moments are connected with flows that are asymmetric with respect to the symmetry plane of the model. In order to examine this more closely all components of the velocity have been determined by SPIV on the lee side of the model in planes perpendicular to the freestream velocity at chordwise positions $\Delta x / c=0.1$ apart from each other. Examples with the notch facing the flow are shown in Fig. 10. At the considered angles of attack vortices are present above both wing halves. The first two cases (Fig. 10a/b) correspond with conditions where no appreciable rolling moment is observed (see Fig. 9a). In both cases the freestream velocity is $u_{\infty}=30 \mathrm{~m} / \mathrm{s}$. In Fig. 10a $\left(\varphi=0^{\circ}, \alpha=10^{\circ}, x / c=0.5\right)$ the flow field looks symmetric, in the other example $(\varphi=$ $90^{\circ}, \alpha=14^{\circ}, x / c=0.8$, Fig. 10b) the velocity field is slightly asymmetric. Hence, slight asymmetries in the flow field do not necessarily serve as a sufficient evidence for the existence of appreciable rolling moments. Fig. 10c relates to the same conditions as the preceding Fig. 10b, except for the freestream velocity that has been increased from $u_{\infty}=30 \mathrm{~m} / \mathrm{s}$ to $u_{\infty}=50 \mathrm{~m} / \mathrm{s}$ where a rolling moment is clearly present (see Fig. $9 \mathrm{c}$ ). At first glance, Fig. $10 \mathrm{~b}$ and $10 \mathrm{c}$ look similar. However, a close look reveals that in the latter case a second vortex is formed on the left wing half, between the leading edge and the primary vortex. In contrast to classical secondary vortices of delta wings its vorticity has the same sign as the primary vortex. Fig. 11 shows another example of such a corotating second vortex in an enlarged section. This case corresponds again with conditions where a rolling moment is clearly present $\left(\varphi=45^{\circ}, \alpha=16^{\circ}, x / c=0.8, u_{\infty}=30 \mathrm{~m} / \mathrm{s}\right.$, see Fig. 9a). Additional vortex lift produced by the second vortex on the left wing half results in a rolling moment of the same sign as observed in the force and moment measurements. Hence, the appearance of a second vortex is related to the generation of a rolling moment. The additional lift of the second vortex acts at a location behind the moment reference point. Therefore it also causes a slight reduction of the pitching moment of up to $\Delta c_{m} \approx 0.01$ (see Fig. $9 \mathrm{~b}$ ). Below, it will be shown that the new vortex is caused by the transition from a leading edge with notch to a round leading edge at the end of the rotatable cylinder. Note that in the present case the origin of two co-rotating vortices of same sign is different from that of other dual vortex structures that have also been observed on non-slender delta wings (Gursul et al., 2005, Luckring and Hummel, 2013).

In Fig. 10b/c, the velocity in the vortex cores is smaller than the freestream velocity. This indicates that the vortices are broken down. On delta wings asymmetric flows can also be caused by different locations of vortex breakdown on the two wing halves. This results in different contributions to vortex lift and, thus, in a rolling moment. Positions of vortex breakdown have been extracted from velocity fields. Here, vortex breakdown is 
considered to be present as soon as the velocity within the vortex drops below the freestream velocity. Note that vortex breakdown is an unsteady phenomenon and PIV results have been averaged over at least 50 double images. Therefore, the error in the position of breakdown is large, of the order of the difference between neighboring PIV planes $(\Delta x / c= \pm 0.1)$. In Fig. 12, positions of vortex breakdown thus determined are plotted $v s$. the angle of attack for $u_{\infty}=30 \mathrm{~m} / \mathrm{s}(x / c=0$ : apex, $x / c=1$ : trailing edge $)$. The baseline configuration $\left(\varphi=180^{\circ}\right)$ is compared with the most effective orientation of the notch $\left(\varphi=45^{\circ}\right)$. For the baseline configuration vortex breakdown happens at the same position on both wing halves (hence there is only one line for $\varphi=180^{\circ}$ in Fig. 12). In contrast, when $\varphi=45^{\circ}$ the left and right vortex break down at different positions - at least at low and intermediate angles of attack. Breakdown on the left wing half happens farther downstream than on the right wing half. This unbalance results in differences in vortex lift that agree with the positive sign of the rolling moment observed under same conditions (see Fig. 9a). On the left wing half with modified leading edge, vortex breakdown is delayed. Note, that on the right wing half the position of vortex breakdown is also different from that of the baseline configuration thus indicating a mutual influence of the flow on the two halves of the delta wing. A comparison of Fig. 9a and 12 shows that intervals of angles of attack in which a rolling moment and different positions of vortex breakdown are observed, overlap but do not fully coincide. Hence, unbalanced vortex breakdown contributes to the generation of a rolling moment but will not be the only cause.

An overview of vortical flow fields on the lee side of the model has been obtained from skin friction lines visualized by means of the oil-film method. In order to show reattachment lines of primary vortices and separation lines of secondary vortices more clearly, these have been extracted form photographs such as Fig. 13a and reproduced graphically as in Fig. 13b. The case considered in Fig. 13 is the same as in Fig. 11, i.e., the corresponding rolling moment is large $\left(\varphi=45^{\circ}, u_{\infty}=30 \mathrm{~m} / \mathrm{s}, \alpha=16^{\circ}\right)$. For checking symmetry, the reattachment line of the primary vortex and the secondary separation line on the right wing half have been mirrored and replotted on the left wing half in Fig. 13b. Close to the apex skin friction structures of the left wing half are difficult to discern but they appear to be slightly different from those of the right wing half. Otherwise, primary reattachment lines agree quite well. Differences are present in secondary separation lines. In the front part of the wing the secondary separation line of the left wing half lies closer to the leading edge (that contains the notch) than on the right wing half. More importantly, there is a qualitative difference between left and right wing half: a new vortex is formed near the tip of the left wing half. This is qualitatively similar to the case of the protruded bar (see above, Fig. 8). The reattachment line of the new vortex sheet begins at the leading edge at a location near the end of the cylinder with notch, and then moves inward. This vortex induces another secondary 
separation. The corresponding line also moves inward in downstream direction. The new vortex pushes the primary vortex, and thus also the secondary separation line induced by the primary vortex, inward. Locations of separation and reattachment lines relating to the new downstream co-rotating vortex are visible in the skin friction lines (Fig. 13 a/b) and can also be inferred from the velocity field (Fig. 11). A comparison shows that the locations obtained from Fig. 13a/b and Fig 11, respectively, are close to each other but do not match exactly. Skin friction lines represent the flow field directly at the surface while the velocity field could only be determined in a region slightly above the surface (because of reflections). This explains slight disagreements in the locations.

Oil-film images have also been taken for other angles of attack and orientations of the notch. In all images relating to conditions that showed an increase of the rolling moment, skin friction lines revealed the formation of an additional co-rotating vortex like in Fig. 13b. Vice versa, no additional vortex was found under conditions where the rolling moment is not increased. This is exemplified in Fig. 13c where separation and reattachment lines obtained from oil-film pictures have been plotted on top of each other within one figure for different orientations of the notch. Flow conditions as in Fig. 9a are considered, at an angle of attack of $\alpha=16^{\circ}$. Red lines correspond with conditions where the rolling moment is increased, blue lines with those of negligible impact on the rolling moment and black lines with the base line configuration. A rolling moment is observed if and only if a second co-rotating vortex is formed at the end of the cylinder with notch. The coincidence of the two phenomena strongly suggests that it is mainly the additional vortex lift co-rotatingon the left wing half that causes a positive rolling moment.

\section{Thin delta wing model with various leading edge geometries}

The thin delta wing model has been tested with leading edges whose shapes can vary between sharp and round along their length. On each wing half the leading edge is divided into two parts, an inner and outer one that can be of different shape. The objective is to generate a lateral force and lateral moments by considering configurations that are asymmetric with respect to the symmetry plane of the model. One wing half has either a completely round or sharp leading edge. The leading edge of the other wing half has the same shape at the apex and an opposing shape at the outer part. A continuous transition between round and sharp parts is denoted, for example, by RSSS. When the transition is abrupt, the labeling is R|SSS, i.e., it includes a bar. Remember, R and $\mathrm{S}$ stand for a round and a sharp part of the leading edge, respectively, and the listing starts at the port side wing tip. In contrast to the actuator model, not only one but two fully symmetric sequences of leading edge shapes can serve as baseline configurations: RRRR and SRRS for SRRR or S|RRR, and SSSS and RSSR for RSSS or 
R|SSS. (Note that an abrupt transition of the leading edge has only been applied on the port side.) Finally, the effect on forces and moments of an asymmetric transition fixing on the upper surface of the thin delta wing model with round leading edges has been addressed. In the following, results of these investigations are again presented for a free stream velocity of $u_{\infty}=30 \mathrm{~m} / \mathrm{s}$.

\subsection{Asymmetric leading edges}

Let us first consider continuous transitions between different shapes of the leading edge. The dependence on the angle of attack of the longitudinal coefficients of various symmetric and asymmetric configurations is reproduced in Fig. 14. In addition, results for a configuration (RR|SS) whose leading edge is entirely round on the port side and completely sharp on the starboard side are included for comparison. Note, that in this latter configuration the transition between round and sharp has been chosen to be abrupt in order to keep the apex pointed. An inspection of Fig. 14 shows that the results can be classed into two groups. It is the shape at the apex that determines the behavior of the longitudinal forces and moment. With a round leading edge at the apex longitudinal coefficients are always close to those of the fully round configuration (RRRR) even if the shape at the wing tips is sharp on one (RRRS) or both sides (SRRS). Similarly, with a sharp leading edge at the apex longitudinal coefficients resemble those of the fully sharp configuration (SSSS) also for configurations with round leading edges at the wing tips (RSSS, RSSR). In particular, features typical of delta wings with fully round and sharp leading edges, respectively, are recovered if only the leading edge at the apex is round or sharp. This includes the onset of stall at smaller angles of attack as well as an increased drag and pitching moment with sharp leading edges. These observations also hold for configurations with abrupt changes in the leading edge contour (R|SSS, S|RRR, not included in Fig. 14). A clear separation of coefficients relating to configurations with sharp and round leading edges at the apex is perhaps somewhat surprising, since differences between round and sharp configurations are partly traced to a suction force acting at round leading edges. With partly round leading edges one might expect a more gradual transition between the limiting cases of fully sharp or round edges. According to Fig. 14, the contribution of the apex region is dominant. The importance of the apex region has also been noted by Lowson and Riley (1995) albeit in a somewhat different context. These authors used an apex flap to control the location of vortex breakdown on a symmetric delta wing with sharp leading edges. Since the vorticity produced at the apex forms the center of the vortex cores, they suggest that changing this vorticity by an apex flap can be used to control the location of vortex breakdown on a delta wing.

In Fig. 15 the dependence on the angle of attack of the lateral coefficients is considered. For symmetric configurations these coefficients should stay at zero and therefore only those of configurations RRRR and SSSS 
are shown for reference. In contrast, all asymmetric configurations, including those with abrupt changes in leading edge shape, are compared with each other. All these cases show a distinct side force and yawing moment, and also a more complex effect on the rolling moment. Except for configuration R|SSS the magnitude of the side force increases with the angle of attack up to the respective onset of stall (see Fig. 15a). Its sign agrees with the direction of the $y$-component of the extra suction force acting on the respective additional round leading edge (negative for configurations R|SSS, RSSS with an additional round part at the outer left leading edge and, similarly, positive for configurations SRRR and $S \mid R R R)$. The extra side force acts mainly behind the moment reference point $\left(x_{N 25}\right)$. This determines the sign of the yawing moment (see Fig. 15b). At low angles of attack the magnitude of the corresponding yawing moment coefficients increases strongly but then quickly levels off. Exceptions are configurations R|SSS and RR|SS. These are addressed below.

The side force of configuration RR|SS is negative, corresponding to a suction force at the round leading edge on the port side. Its absolute value is roughly twice that of any of the other asymmetric configurations. In these latter cases the extra side force acts only at the outer part of the wing while with configuration RR/SS a suction force acts along the whole leading edge whose length is about twice that of the former cases thus explaining the approximately doubled force. Note that the magnitude of the side force begins to decrease prior to stall. The yawing moment of the configuration RR|SS exhibits a more complex behavior. Initially, at small angles of attack, it increases in the same manner as the other asymmetric configurations. This hints at a prevailing influence of the rear part of the round leading edge of configuration RR|SS. With increasing angle of attack the leading-edge separation progresses upstream, from the wing tip towards the apex. Hence, suction forces acting at the front part of the round leading edge become dominant. Note that the front part of the leading edge is located ahead of the moment reference point. Eventually, this leads to a reversal of the sign of the yawing moment. However, at even greater angles of attack, where the magnitude of the side force begins to decrease, the course of the yawing moment coefficient is changed once again. The reason for this complex behavior is not fully clear.

All asymmetric configurations exhibit a rolling moment although within different ranges of the angle of attack (see Fig. 150). A negative sign implies that the lift of the right wing half exceeds that of the left wing half and vice versa. In all cases considered, the fraction of the sharp leading edge is greater on the wing half with enhanced lift. Vortices formed at sharp leading edges are stronger than those formed at round ones (Luckring, 2010), thus contributing to a greater vortex lift on the respective wing half. 
Vortices originating from the leading edges of the two wing halves of a delta wing interact with each other. This mutual interaction is exemplified in Fig. 16. Velocity fields are compared with each other in a plane perpendicular to the free stream at location $x / c=0.31$, i.e., slightly upstream of possible changes in the leading edge shape. The angle of attack is $\alpha=20^{\circ}$ and the free stream velocity is again $u_{\infty}=30 \mathrm{~m} / \mathrm{s}$. The configurations with fully round and sharp leading edges (Fig. 16a/b) show well-known behaviors like a further inboard location and an earlier breakdown of vortices formed at sharp leading edges. However, this no longer holds for the configuration RR|SS. Here, vortices of the left and right wing half obviously exert an influence on each other (see Fig. 16c). The vortex of the round leading edge is already broken down while that of the sharp leading edge is still almost fully intact, only near its axis the velocity begins to be reduced. Apparently, a displacement effect due to the larger vortex originating from the sharp leading edge causes an earlier breakdown of the smaller vortex from the round leading edge. Similarly, since the smaller vortex of the round leading edge causes a reduced displacement this results in a downstream shifted breakdown of the vortex originating at the sharp leading edge. Likewise, there is an upstream effect of the downstream parts of the leading edges. With sharp leading edges at the downstream and round ones at the upstream part of the wing, vortices of the symmetric configuration SRRS are broken down at the location $x / c=0.31$ (see Fig. 16d). This is in contrast to the performance of configuration RRRR but similar to that of the left, vortex of configuration RR|SS. Similarly, at $x / c=0.31$ the vortex on the right wing half of configuration RRRS is already broken down while that on the left wing half with a fully round leading edge is not (see Fig. 16e). With sharp leading edges at the apex and round ones at the rear part the effect is more subtle, for example, there is little difference between the flow fields of configuration RSSR (Fig. 16f) and configuration SSSS (Fig. 16b).

As discussed above, vortex effects are also reflected in velocity profiles. In Fig. 17, the relative velocity magnitude is plotted versus the spanwise direction at a height above the left wing half that corresponds with the location of the respective vortex cores of different configurations. Note that the chordwise position of the profiles is the same as in the previous figure but the angle of attack is slightly lower, $\alpha=17.5^{\circ}$. When a configuration with fully sharp left leading edge is changed from SSSS to SS|RR the conversion of a left fully broken down vortex into one with incipient breakdown is well reproduced. For a fully round left leading edge differences due to changing the right hand side from round to sharp are less pronounced. This is likely because the vortex formed by a shear layer separating from a round leading edge is of lesser strength so that differences are less striking. 
An additional co-rotating primary vortex can be formed behind a change in the geometry of a leading edge. This has already been shown in the preceding section on the actuator model. There, a new vortex appeared downstream of the end of the actuator when the actuator is applied (see Fig. 13). The abrupt interruption of the leading-edge contour initiates the formation of an additional downstream vortex that is co-rotating with the leading-edge vortex. In order to check whether additional leading-edge vortices are also formed in the case of the thin model with both, abrupt and smooth changes in shape along the leading edge, again primary reattachment and secondary separation lines have been taken from oil-film images and sketched at different angles of attack. Examples obtained for configurations R|SSS and RSSS are compared with each other in Fig. 18 at $\alpha=8^{\circ}$ and $\alpha=16^{\circ}$. In both configurations an additional primary vortex is formed at the outer round part of the left leading edge when the angle of attack is small $\left(\alpha=8^{\circ}\right)$. However, at the greater angel $\left(\alpha=16^{\circ}\right)$ a second primary vortex is only observed with configuration R|SSS, i.e., when the transition between the different leading edge shapes happens abruptly. When the transition is continuous (RSSS) only a single primary vortex is present on each wing half.

In general, at leading edges with a change in geometry two primary vortices are formed at small angles of attack while only one is formed at greater angles. The limiting angle below which a second primary vortex is obtained depends on the particular configuration. For configurations S|RRR, SRRR and RSSS it is roughly $\alpha=$ $10^{\circ}$, and for $\mathrm{R} \mid \mathrm{SSS}$ it is approximately $\alpha=18^{\circ}$. In the latter case, where the transition from sharp at the apex to round at the wing tip is sudden, the primary separation line at the rounded outer leading edge lies below that of the sharp leading edge when the angle of attack is large. Supposably, this enables the formation of a second primary vortex up to greater angles of attack.

The findings relating to those angle of attack ranges where a second primary vortex is formed near the wing tip explains some of the behavior of the lateral coefficients (see Fig. 15). Rolling moments typically occur at angles of attack at which also a second leading-edge vortex forms downstream of the first one. With configurations R|SSS and RSSS, the additional vortex formed at the round leading edge of the left wing half is comparatively weak and, therefore, lift is greater on the right wing half resulting in a negative rolling moment. Similarly, with configurations S|RRR and SRRR the additional round part of the leading edge is located at the outer part of the right wing half thus reducing its lift. This results in a positive rolling moment. The situation is different for configuration RR|SS. Here, the rolling moment coefficient initially remains small but becomes negative at greater angles of attack. This is probably connected with an earlier occurrence of vortex breakdown, and thus drop in lift, on the left wing half with round leading edge (see Fig. 16c). 
Fig. $15 \mathrm{~b}$ shows that the absolute value of the yawing moment initially increases strongly with angle of attack for all configurations. The corresponding angle range coincides with the occurrence of a second primary vortex. Note that this also holds for configuration R|SSS (here, the second co-rotating vortex disappears at $\alpha \approx 18^{\circ}$ ). However, the increase of the absolute yawing moment usually ends somewhat before the second (downstream) leading-edge vortex disappears. As mentioned above, the side force of most asymmetric configurations reaches its extreme values when the wing stalls. An exception is configuration R|SSS that reaches the extreme value of the side force at lower angles of attack than the onset of stall. In this case, the corresponding angle, $\alpha \approx 18^{\circ}$, is the angle at which the second co-rotating vortex disappears.

\subsection{Asymmetric transition tripping}

The way vortices are formed and, thus, the location of primary reattachment and secondary separation lines depends on the state of the boundary layer (Hummel, 2004). In wind-tunnel tests, a forced laminar-turbulent transition of the boundary layer is achieved by applying turbulence generators near the leading edge. Since turbulent boundary layers can withstand adverse pressure gradients better than laminar ones, the onset of flow separation is delayed. In the present case of delta wings, the onset of leading-edge flow separation and the subsequent formation of vortices are shifted downstream. Hövelmann et al. (2016) and Buzica et al. (2018) have experimentally studied the effect of different tripping devices on the flow about a diamond wing of the same leading-edge sweep as that of the present delta wings. As compared to free transition, the pitching moment decreases as long as the tripping devices are not too high. They attribute this to a changed lift distribution on the suction side of the wing, caused by the more downstream formation of the vortices. Similarly, drag decreases due to leading-edge suction at the upstream part of the leading edge where the flow is still attached. In contrast, according to Hövelmann et al. (2016), over-tripping by too large tripping devices results in a premature onset of flow separation and vortex formation. This causes an increase of vortex lift in the apex region, i.e., upstream of the moment reference point and, thus, an increase of the pitching moment as compared to free transition.

In the present study, the effect of asymmetric transition tripping on the lateral force and lateral moments has been explored in a controlled way for configuration RRRR with fully round leading edges. The boundary layer of the left (port side) wing half has been tripped by a row of trip dots. Three different arrangements have been tested: tripping applied along the whole length of the left leading edge (version TR 1 ), tripping from the apex to the middle of the leading edge (version TR2), and finally tripping from the middle of the leading edge to the wing tip (version TR3). If applied trip dots were placed very close to the leading edge, with the center of the dots lying about $1 \mathrm{~mm}$ (approximately one diameter of a dot) behind the leading edge, i.e., they still lie on the 
ellipsoidal part of the leading edge. With a fully tripped left leading edge (version TR1) the pitching moment was less than that of the clean wing. This indicates that the boundary layer was not over-tripped.

One-sided tripping has an effect on the lateral force and the yawing moment in a small interval of the angle of attack (see Fig. 19). The effect on the rolling moment is smaller and less distinct. Tripping at the leading edge generates high suction peaks (Ashill et al., 1990). Here, the side force becomes negative corresponding with an extra suction force at the tripped leading edge. The suction force causes a positive yawing moment. Hence, in all cases considered the contribution to yawing of suction near the apex which results in a negative yawing moment, is outbalanced by the greater lever arm of the suction force at the rear part of the leading edge.

An examination of reattachment and secondary separation lines of vortices, again taken from oil-film images, shows that with tripping regions of attached flow are present as long as the angle of attack is low. An example is shown in Fig. 20a for $\alpha=8^{\circ}$ where lateral effects occur. Qualitatively, oil-film images show the same features also at smaller angles of attack where no effect is present in the lateral force and moments. A primary and secondary vortex is formed at the front part of the wing if only the rear part of the leading edge is tripped (version TR3) and vice versa (TR2), and no signs of yortices are present on the left wing half when the whole leading edge is tripped (TR1). With increasing angle of attack the primary separation line moves to the lower surface of the wing, away from the trip dots. The suppressing effect of tripping on primary separation and vortex formation decreases. Already at $\alpha=10^{\circ}$ a vortex is formed for version TR1 (Fig. 20b). With further increasing angle of attack, vortices on the two wing halfs accordingly become symmetric with respect to the symmetry plane of the model.

With version TR3 a primary vortex is formed by shear layer separation at the front non-tripped part of the leading edge. The vortex continues over the rear part but is no longer fed there. Therefore, signs of the primary reattachment and also secondary separation lines are phasing out in the downstream region and are no longer discernable in the original oil-flow image corresponding with Fig. 20. It is conjectured that the presence of the vortex influences the flow around the rear part of the leading edge in such a manner that leading edge suction becomes reduced thus explaining the reduction in side force as compared to the other two versions (TR1 and TR2, see Fig. 19a). Since, howeyer, the lever arm is greater when suction acts further back than further ahead resulting yawing moments of version TR2 and TR3 can be similar as is observed in Fig. 19b. At small angles, below those where lateral effects are noticeable in forces and moments, footprints of vortices are qualitatively similar to those shown in Fig. 20. However, since vortices are just beginning to be formed their effect and also that of possible suction forces are too small to be seen in the lateral coefficients. 


\section{Conclusion}

Concepts of flow control investigated in the past often aimed at improving aerodynamic characteristics of delta wings such as lift and drag, particularly, in the post stall regime. The focus of the present paper is on increasing the lateral maneuverability of delta wings. Various concepts based on measures applied at the leading edge were examined in wind tunnel tests. One group of concepts is based on static and dynamic actuators, the other on the idea that morphing wings could be used to change leading-edge shapes. Both/approaches are applied in such a way that the shape of the leading edge becomes asymmetric with respect to the symmetry plane of the model. This results in the formation of asymmetric delta wing vortices and thus lateral moments. The assessment of the concepts by measuring forces and moments has shown that lateral moments that are sufficient for control can be generated. Analyses of the flow field by means of PIV and oil-flow visualization of skin friction lines hint at a dominant influence of a new vortex formed at the rear part of the leading edge, either behind the end of the actuator or behind the transition between different leading edge shapes.

The actuator model with a protruding bar provides a well-defined location of flow separation. With a bar applied on one wing half a rolling moment is generated that depends in a non-uniform way on the angle of attack. For the purpose of flow control this is not applicable. However, an unsteady forcing of the separating shear layer by the oscillating bar showed a maximum effect at a Strouhal number of about one. Thus, an unsteady forcing of the separating shear layer appears generally promising.

The leading-edge cylinder with a notch aligned parallel to its axis allows for appropriately positioning the notch by turning the cylinder. The appropriate position depends on the velocity (Reynolds number) and ensures a non-zero rolling moment within a significant range of angles of attack. Positive rolling moments correlate with a decrease in the pitching moment. This also holds for the protruding bar actuator. In both cases a second corotating leading-edge vortex forms behind the end of the actuator near the tip of the delta wing. With the notched cylinder the second co-rotating vortex was always observed when a rolling moment occurred. Obviously it is the new vortex that provides additional vortex lift causing a positive rolling moment and a decrease in the pitching moment. Furthermore, a delay of vortex breakdown of the upstream primary vortex occurred under same conditions as the rolling moment. The delay may be due to a stabilizing effect of the additional (downstream) co-rotating vortex similar to double delta wing configurations.

The effect of different leading edge shapes has been investigated on the thin model. In symmetric arrangements of different shapes along the left and right leading edge, the shape at the apex controls both, longitudinal forces and the pitching moment. Characteristic features of fully round and sharp leading edges, respectively, are recovered if only the shape at the apex is round or sharp. Consequently, the leading edge 
geometry near the apex dominates the aerodynamic behavior. In asymmetric arrangements, round segments are longer on one wing half than on the other. Up to the onset of stall, the suction force acting on the additional round segment causes a lateral force and moments. The yawing moment increases with angle of attack as long as a second primary vortex forms at the leading edge shape transition. Thereafter it remains approximately constant.

Vortices above the two wing halves of a delta wing exert an influence on each other. If leading edges of the thin model with uniform and same shapes on both wing halves, are changed into an asymmetrical arrangement by exchanging a leading edge segment on one wing half only, also the flow field on the unmodified wing half is affected. In particular, a shift of the position of vortex breakdown has been observed on the unmodified wing half. A mutual interaction of vortices has also been observed on the actuator model with notched cylinder (see Fig. 12). This mutual interaction of vortices needs to be considered in wind tunnel tests, for example, when lateral effects of control surfaces are investigated using half models. Finally, there is an upstream effect of leading edge shapes both with symmetric and asymmetric arrangements. If, for example, the rear part of a fully round leading edge is changed into sharp one the position of vortex breakdown is shifted upstream up to a position where the leading edge is still round.

A one-sided delay of separation at the leading edge by transition tripping results in asymmetric flows that generate side forces and yawing moments. Within the limits tested the effect was relatively weak. The range of possible applications can be extended by more effective concepts of separation delay as, for example, a dynamic surface roughness (Huebsch, 2006).

The concepts of flow control for delta wings explored in this paper can be grouped into different techniques of longitudinal vortex control as distinguished by Bushnell (1992). Separation control has been applied with stationary actuators and boundary layer tripping at the leading edge. The oscillating bar showed a potential for altering the stability of vortices. A change in leading edge geometry at the end of actuators or at the transition between different leading edge shapes can result in the formation of a new vortex at the rear part of the leading edge. This can be considered as segmentation of the primary delta wing vortex. The second primary vortex provides vortex lift near the wing tip. This makes the lever arm large and the effect on the rolling moment big. Finally, the new vortex near the tip can be considered a control vortex that stabilizes the original primary vortex. 


\section{References}

Ashill, P. R., Fulker, J. L., Simmons, M. J., Betts, C. J., "Flow features of highly swept wings at subsonic and supersonic speeds," ICAS-90-3.9.1, 1678-1689, 1990

Azuma, D., Nakamura, Y., "Lift Enhancement of a Thick Delta Wing Using Rotational Leading-Edges," AIAA 2002-0847, 40th AIAA Aerospace Sciences Meeting \& Exhibit, 14-17 Jan 2002, Reno, NV, USA, 2002

Bushnell, D. M. "Longitudinal vortex control - techniques and applications," The Aeronautical J. 96(958), 1992

Busse, F., "Experimentelle Untersuchung von Maßnahmen der Strömungssteuerung und des Einflusses der Transition an Vorderkanten schwach gepfeilter Delta-Flügel," DLR-IB 224-2013 A 86, DLR, Germany, 2013

Buzica, A., Debschütz, L., Knoth, F., Breitsamter, C., "Leading-Edge Roughness Affecting Diamond-Wing Aerodynamic Characteristics," Aerospace 5(3), 2018

Chen, H., Wang, J.-J., "Vortex structures for flow over a delta wing with sinusoidal leading edge" Exp. Fluids 55, 1761, 2014

Deng, Q., Gursul, I., "Vortex breakdown over a delta wing with oscillating leading edge flaps," Experiments in Fluids 23 , 347-352, 1997

Edzards, F., "Wind tunnel investigations to estimate the efficiency of modified leading edges as a means of flow control on a delta wing," Bachelor Thesis, University of Göttingen, 2014

Erickson, G. E., "Wind Tunnel Investigation of Passive Porosity Applied to the Leading-Edge Extension and Leading-Edge Flaps on a Slender Wing at Subsonic Speeds," NASA/TM-2017-219596, Hampton, VA, 2017

Frith, S. P., Wood, N. J., "Use of Circulation Control for Flight Control," in: G. S. Jones, R. D. Joslin, "Applications of Circulation Control Technology," American Institute of Aeronautics and Astronautics, Reston, VA, 337-353, 2006

Furman, A., Breitsamter, C., "Turbulent and unsteady flow characteristics of delta wind vortex systems," Aerospace Science and Technology 24, 32-44, 2013

Gonuney, T., Rockwell, D., "Flow past a delta wing with a sinusoidal leading edge: near surface topology and flow structure," Exp.Fluids 47, 321-331, 2009

Greenblatt, D., Kastantin, Y., Nayeri, C. N., Paschereit, C. O., "Delta-Wing Flow Control Using Dielectric Barrier Discharge," AIAA J. 46(6), 1554-1560, 2008

Gursul, I., Gordnier, R., Visbal, M., "Unsteady aerodynamics of nonslender delta wings," Prog. Aerosp. Sci. 41, 515-557, 2005

Gursul, I., Wang, Z., Vardaki, E., "Review of flow control mechanisms of leading-edge vortices," Prog. Aerosp. Sci. 43, $246-270,2007$

Hövelmann, A., Breitsamter, C., "Leading edge geometry effects on the vortex formation of a diamond wing," J. Aircraft 52(5), 1596-1610, 2015

Hövelmann, A., Knoth, F., Breitsamter, C., "AVT-183 diamond wing flow field characteristics Part 1: Varying leading-edge roughness and the effects on flow separation onset," Acrospace Science and Technology 57, 18-30, 2016

Huang, A., Folk, C., Silva, C., Christensen, B., Chen, Y., Ho, C. M., Jiang, F., Grosjean, C., Tai, Y. C. Lee, G. B., Chen, M., Newbern, "Application of MEMS Devices to Delta Wing Aircraft: From Concept Development to Transonic Flight Test," AIAA 2001-0124, 39th AIAA Aerospace Sciences Meeting \& Exhibit, 8 - 11 Jan 2001, Reno, NV, 2001

Huebsch, W. W., "Two-Dimensional Simulation of Dynamic Surface Roughness for Aerodynamic Flow Control," J. Aircraft 43(2), 353-362, 2006

Hummel, D., "Effects of Boundary Layer Formation on the Vortical Flow above Slender Delta Wings," in: "Enhancement of NATO Military Flight Vehicle Performance by Management of Interacting Boundary Layer Transition and Separation," RTO-MP-AVT-111, Prague, Czech Republic, 4-7 October 2004 (DOI: 10.14339/RTO-MP-AVT-111)

Kaiden, T., Nakamura, Y., "Numerical Analysis of Aerodynamic Control of Delta Wing by Microflap," J. Aircraft 41(3), $555-563,2004$

Karagounis, T., Maxworthy, T., Spedding, G. R., "Generation andControl of Separated Vortices over a Delta Wing by Means of Leading Edge Flaps," AIAA-89-0997, AIAA $2^{\text {nd }}$ Shear Flow Conf., 13-16 March 1989, Tempe, AZ, 1989

Kölzsch, A., "Active Flow Control of Delta Wing Leading-Edge Vortices," Ph.D. Thesis, Techn. Univ. Munich, 2017

Kölzsch, A., Breitsamter. C., "Vortex-Flow Manipulation on a Generic Delta-Wing Configuration," J. Aircraft, 51(5), 13801390, 2014

Lee, G.-B., Shih, C., Tai, Y.-C., Tsao, T., Liu, C., Huang, A., Ho, C.-M., "Robust Vortex Control of a Delta Wing by Distributed Microelectromechanical-Systems Actuators," J. Aircraft, 37 (4), 697-706, 2000

Lowson, M. V., Riley A. J., "Vortex Breakdown Control by Delta Wing Geometry," J. Aircraft 32 (4), 832-838, 1995

Luckring, J. M., "A Survey of Factors Affecting Blunt Leading-Edge Separation for Swept and Semi-Slender Wings," AIAA 2010-4820, $28^{\text {th }}$ AIAA Applied Aerodynamics Conference, 28 June - 1 July 2010, Chicago, IL, USA, 2010

Luckring, J. M., Hummel, D., "What was learned from the new VFE-2 experiments," Aerospace Science and Technology 24(1), 77-88, 2013

Matsuno, T., Yokouchi, S., Kaiden, T, Nakamura, Y., "Flow Control on a 45-Degree Delta Wing Using a Small Flap," AIAA 2002-0558, 40th AIAA Aerospace Sciences Meeting \& Exhibit, 14-17 Jan 2002, Reno, NV, USA, 2002

Menke, M., Yang, H., Gursul, I., "Experiments on the unsteady nature of vortex breakdown over delta wings," Experiments in Fluids 27, 262-272, 1999

Miau, J. J., Kuo, K. T., Liu, W. H., Hsieh, S. J., Chou, J. H., Lin, C. K., "Flow Developments Above 50-Deg Sweep Delta Wings with Different Leading-Edge Profiles," J. Aircraft, 32 (4), 787-794, 1995

Mitchell, A. M., Delery, J., "Research into vortex breakdown control," Prog. Aerosp. Sci. 37, 385-418, 2001

Modi, V. J., "Moving Surface Boundary-Layer Control: A Review," J. Fluid Structures 11, 627-663,1997 
Paul, M., Rein, M., "Transonic Numerical and Experimental Evaluation of Unconventional Lambda Wing Control Surfaces," J. Aircraft 54(3), 1139-1149, 2017, doi: 10.2514/1.C034080

Rao, D. M., "Leading Edge Vortex-Flap Experiments on a 74 Deg Delta Wing," NASA-CR-159161, Hampton, VA, 1979

Rao, D. M., Johnson Jr., T. D., "Investigation of Delta Wing Leading-Edge Devices," J. Aircraft 18, 161-167,1981

Renac, F., Barberis, D., Molton, P., "Control of Vortical Flow over a Rounded Leading-Edge DeltaWing," AIAA Journal, 43(7), 1409-1418, 2005

Siegel, L., "Experimentelle Analyse der Strömung am schwach gepfeilten Deltaflügel mit unterschiedlichen Vorderkantengeometrien unter dem Gesichtspunkt der Strömungssteuerung," DLR-IB 224-2013 A79, DLR, Germany, 2013

Traub, L. W., Galls, S. F., "Effects of Leading- and Trailing-Edge Gurney Flaps on a Delta Wing," J. Aircraft 36 (4), 651658,1999

Vasista, S., Riemenschneider, J., van de Kamp, B., Monner, H. P., Cheung, R. C. M., Wales, C., Cooper, J. E., "Evaluation of a Compliant Droop-Nose Morphing Wing Tip via Experimental Tests," J. Aircraft 54(2), 519-534, 2017

Verhaagen, N. G., "Leading-Edge Radius Effects on Aerodynamic Characteristics of 50-Degree Delta Wings," J. Aircraft 49(2), 521-531, 2012

Wentz Jr., T. Z., Kohlman, D. L., "Vortex Breakdown on Slender Sharp-Edged Wings," J. Aircaft 8(3), 156-161, 1971

Wilke, J. B., "Aerodynamische Strömungssteuerung mittels dielektrischer Barriereentladungs-Plasmaaktuatoren," (Aerodynamic flow-control with dielectric barrier discharge plasma actuators), DLR Forschungsbericht 2009-19, DLR, Germany, 2009

Williams, N. M., Wang, Z., Gursul, I., "Active Flow Control on a Nonslender Delta Wing," J. Aircaft 45(6), 2100-2110, 2008 


\section{Figures}

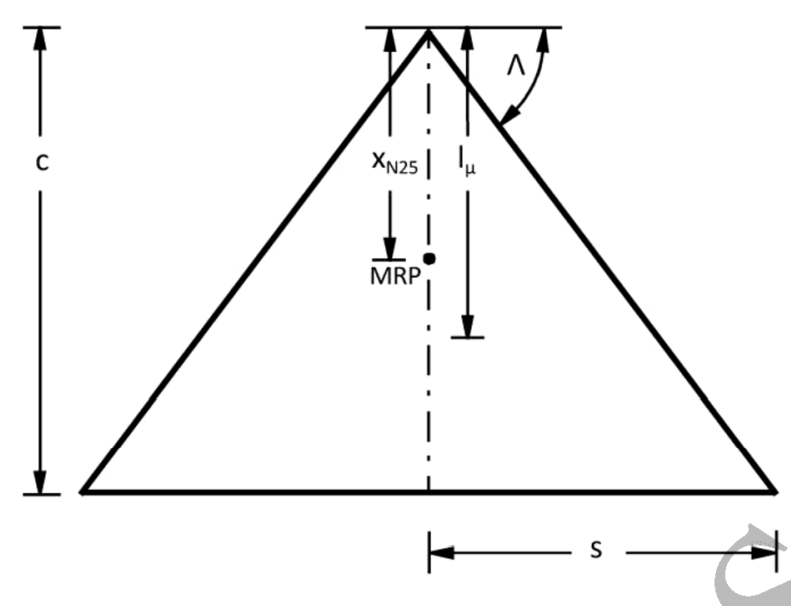

Fig. 1 Wing planform of the delta-wing models: sweep angle $(\Lambda)$, half span (s), root chord (c), aerodynamic mean chord $\left(1_{\mu}\right)$, neutral point $\left(\mathrm{x}_{\mathrm{N} 25}\right)$ and moment reference point (MRP).

a)

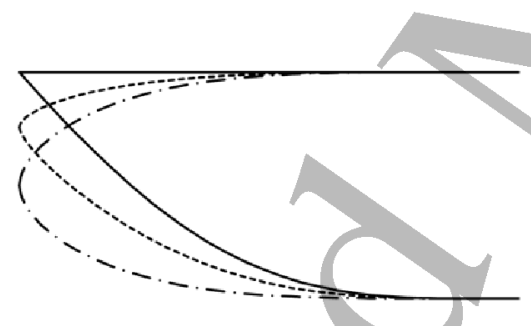

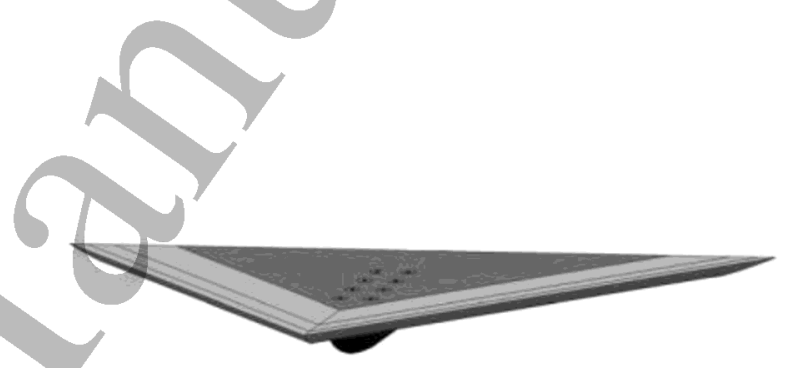
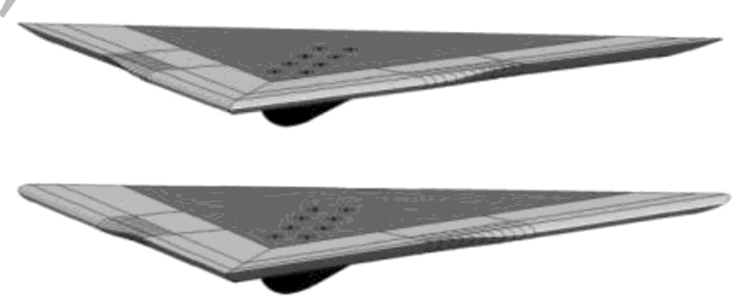

b)

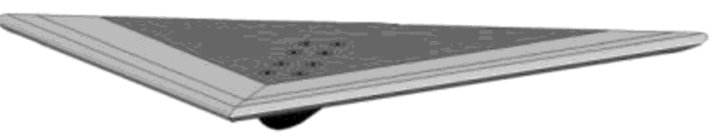

Fig. 2 Thin model with different leading-edge contours:

a) sharp leading edge (solid line), elliptic leading edge (dash-dotted line) and transition between these two (dashed line),

b) different leading-edge combinations, from top to bottom: fully sharp (SSSS), sharp-round-round-sharp (SRRS), round-sharp-sharp-round (RSSR), fully round (RRRR). 
a)

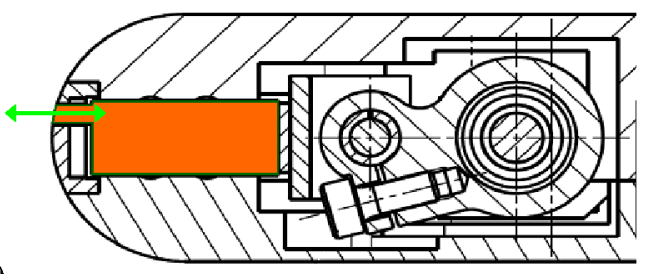

Fig. 3 Actuator model: a) protruding bar, b) cylinder with notch.
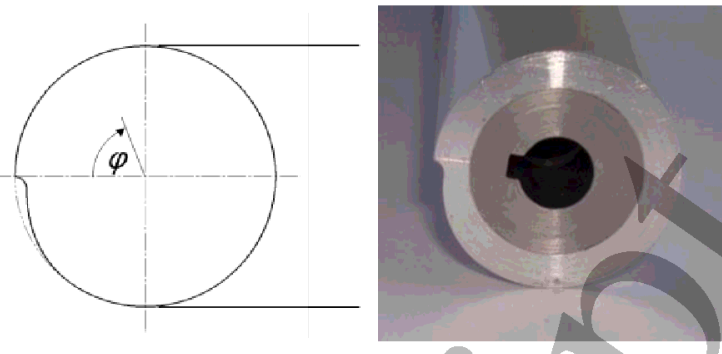

b)

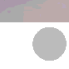

a)
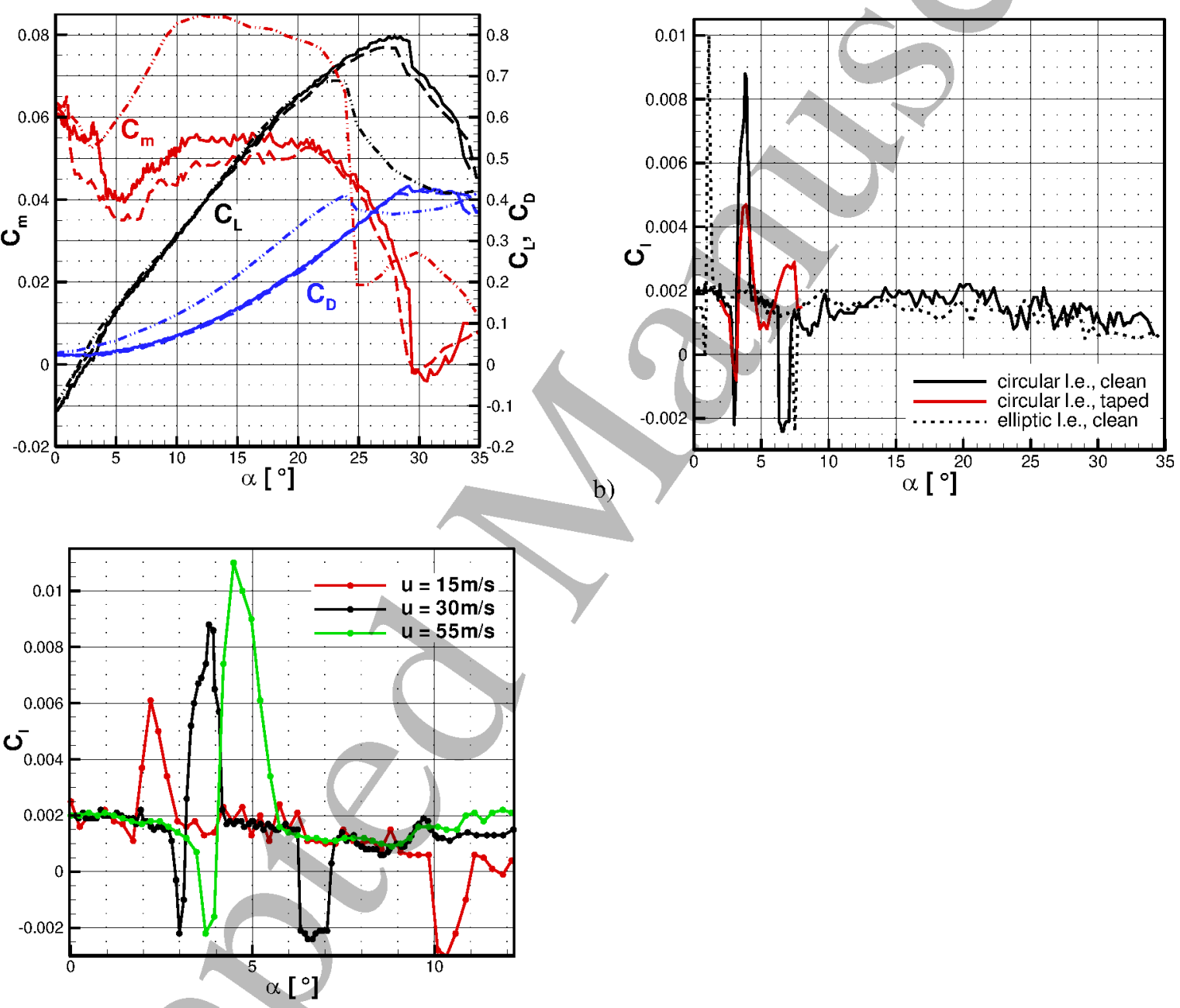

c)

Fig. 4 Thin model: aerodynamic coefficients vs. AoA:

a) lift (black lines), drag (blue lines) and pitching moment (red lines) for circular (solid lines), elliptic (dashed lines) and sharp (dash-dotted lines) leading edges $\left(u_{\infty}=30 \mathrm{~m} / \mathrm{s}\right)$,

b) spikes in rolling moment coefficient (rounded leading edge, $u_{\infty}=30 \mathrm{~m} / \mathrm{s}$ ),

c) effect of velocity on spikes in rolling moment coefficient (circular leading edge). 


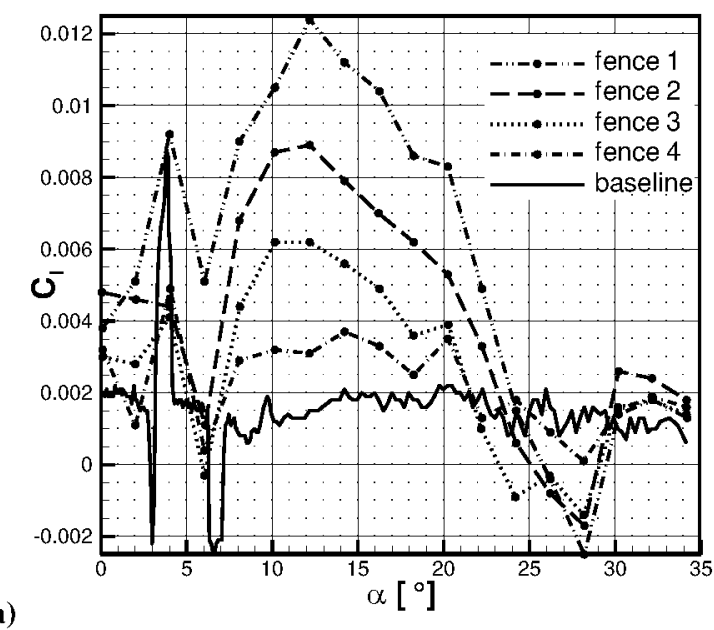

a)

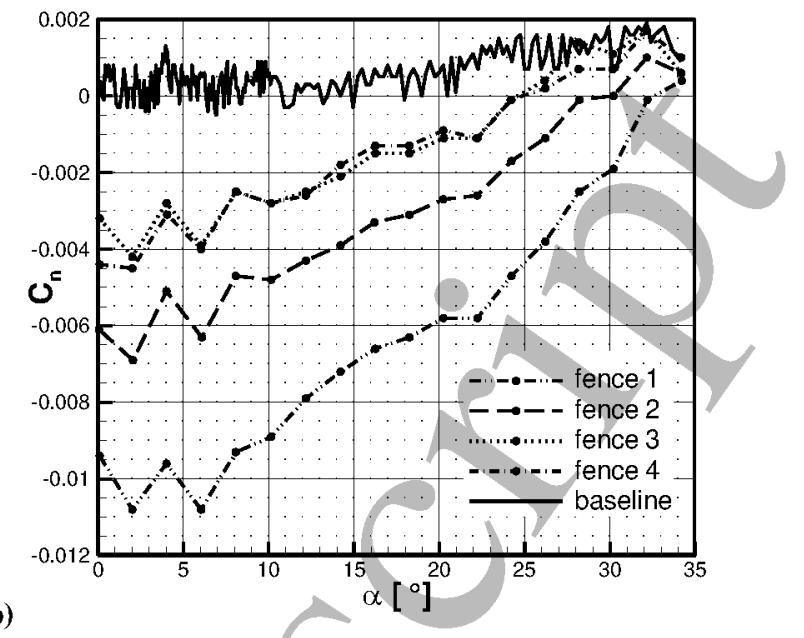

b)

Fig. 5 Thin model with leading edge fences: a) rolling moment coefficient and b) yawing moment coefficient vs. AoA.

Baseline, and fence $1\left(100 \times 10 \mathrm{~mm}^{2}\right.$, at wing tip), fence $2\left(50 \times 10 \mathrm{~mm}^{2}, 50 \mathrm{~mm}\right.$ inward of wing tip), fence 3 $\left(50 \times 5 \mathrm{~mm}^{2}, 50 \mathrm{~mm}\right.$ inward of wing tip), and fence $4\left(50 \times 5 \mathrm{~mm}^{2}\right.$, at wing tip).

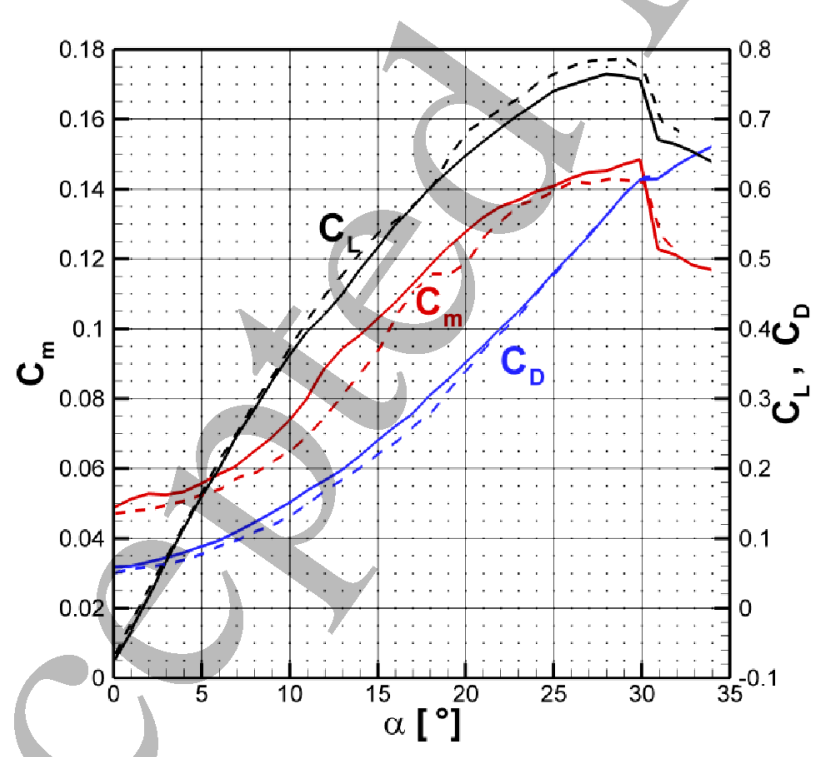

Fig. 6 Actuator model with protruding bar: longitudinal coefficients vs. AoA,

clean (solid lines) and protruding bar (dotted lines), $u_{\infty}=30 \mathrm{~m} / \mathrm{s}$. 


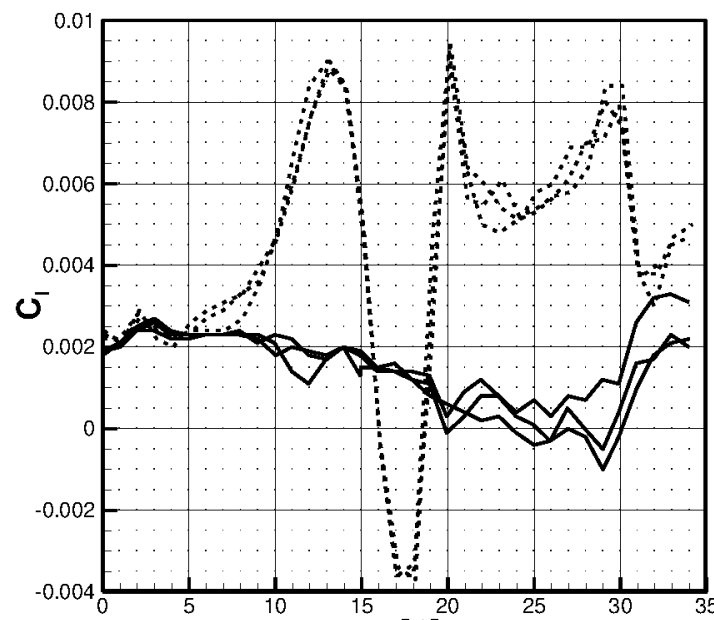

a)

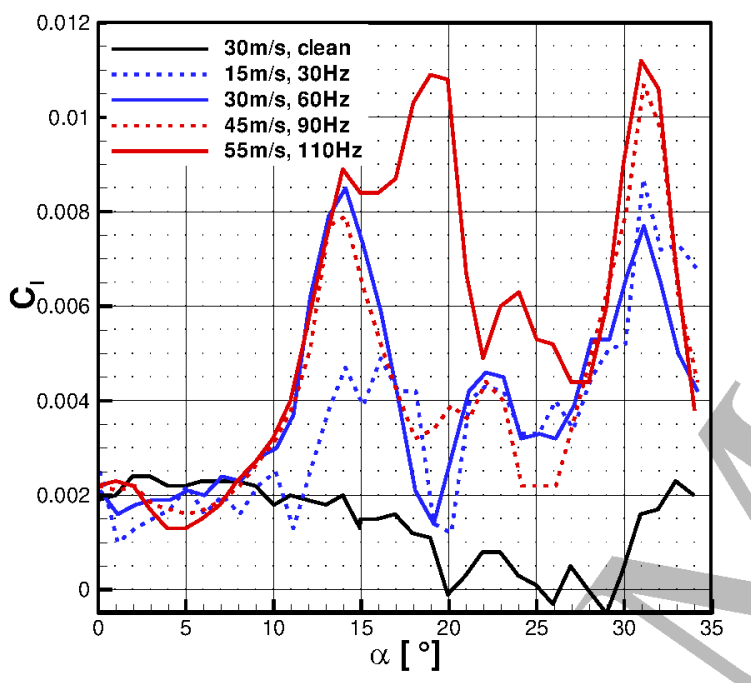

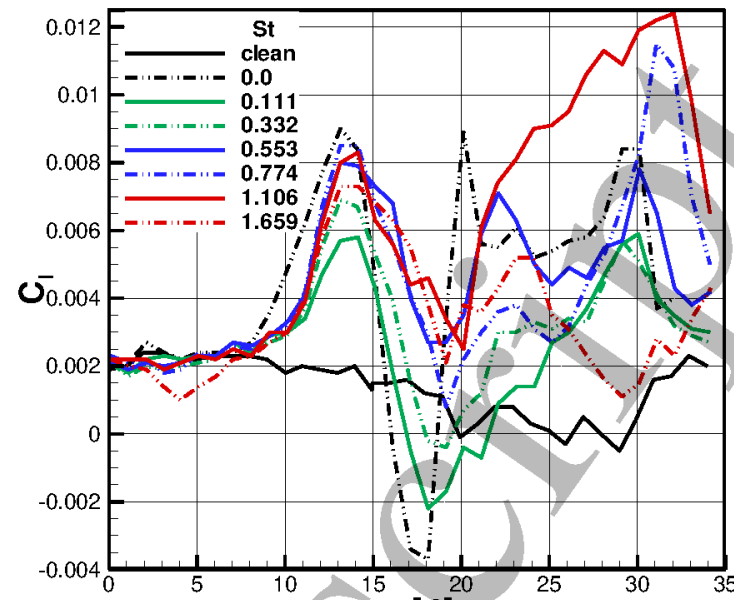

b)

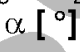

c)

Fig. 7 Actuator model with protruding bar: rolling moment coefficient vs. AoA

a) stationary bar, clean (solid lines) and protruding bar (dotted lines), three repetitions each, $u_{\infty}=30 \mathrm{~m} / \mathrm{s}$,

b) effect of Strouhal number: clean configuration and oscillating bar, $u_{\infty}=30 \mathrm{~m} / \mathrm{s}, \mathrm{St}=f c / u_{\infty}=0.0-1.66$,

c) effect of velocity: clean and oscillating bar, $\mathrm{St}=0.66, u_{00}=15-55 \mathrm{~m} / \mathrm{s}$. 
a)

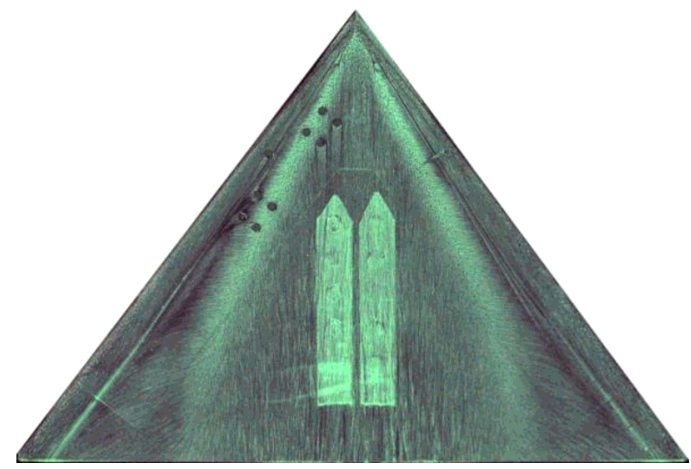

b)

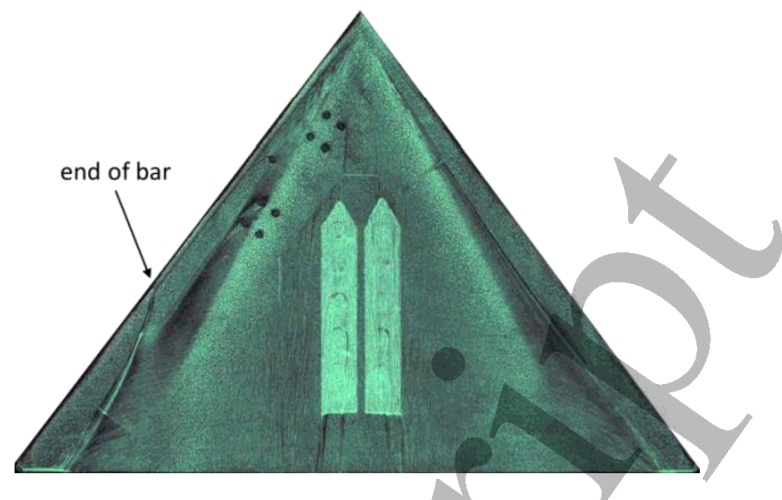

Fig. 8 Oil-flow images of lee side of actuator model $\left(u_{00}=30 \mathrm{~m} / \mathrm{s}, a=14^{\circ}\right)$ : a) clean, b) with protruding bar on left leading edge.

a)
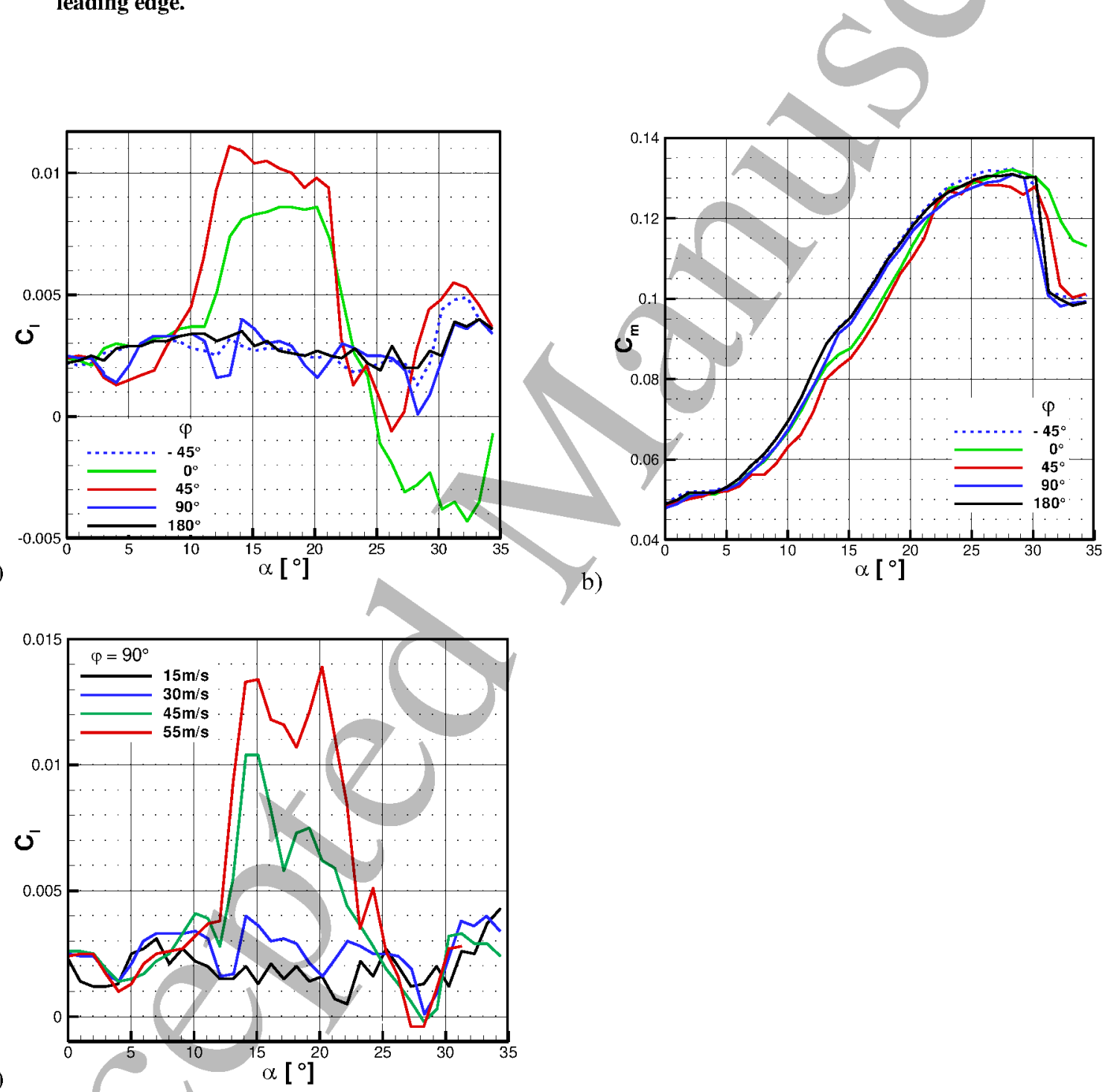

Fig. 9 Actuator model with notched cylinder, rolling and pitching moment coefficients vs. AoA: a) and b) effect of orientation of notch $\left(u_{\infty}=30 \mathrm{~m} / \mathrm{s}\right): \varphi=180^{\circ}$ (clean) and $\varphi=-45^{\circ}, 0^{\circ}, 45^{\circ}$ and $90^{\circ}$, c) effect of velocity on rolling moment coefficient $\left(\varphi=90^{\circ}\right): u_{\infty}=15 \mathrm{~m} / \mathrm{s}-55 \mathrm{~m} / \mathrm{s}$. 
a)

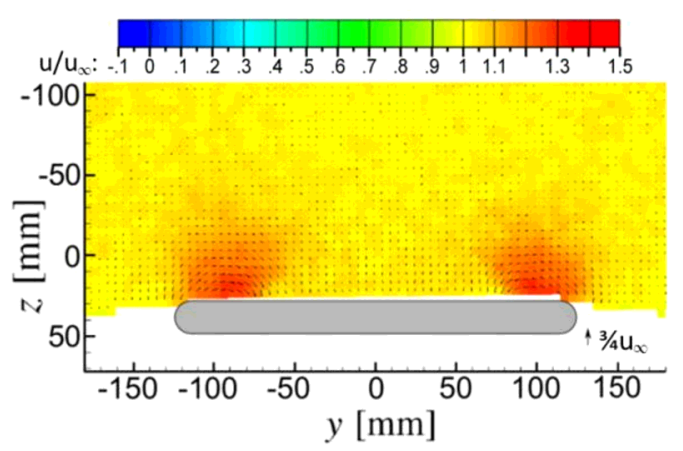

b)

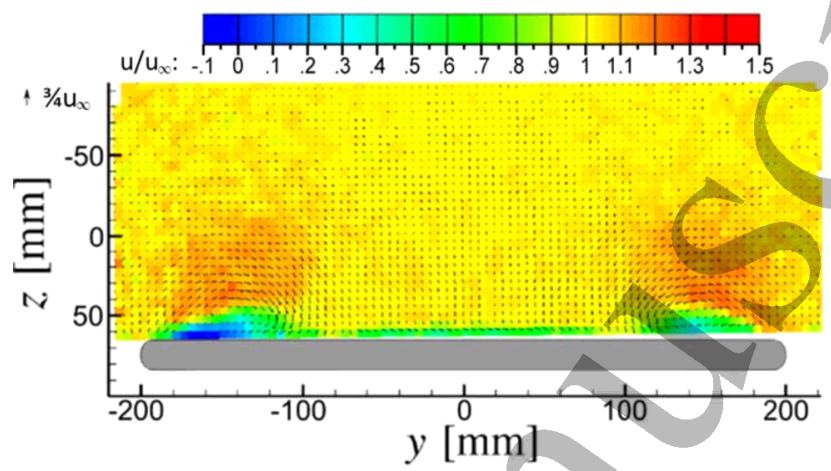

c)

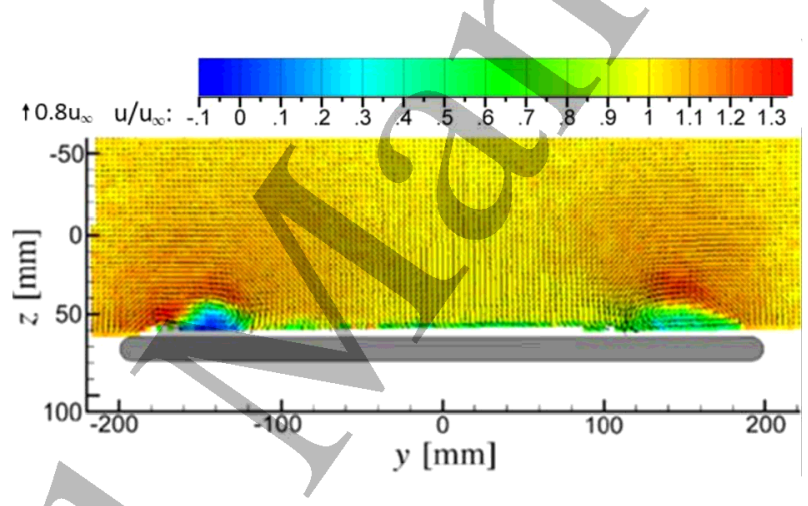

Fig. 10 Actuator model with notched cylinder: velocity fields above model (pilot's view), velocity magnitude colour coded.
a) $u_{\infty}=30 \mathrm{~m} / \mathrm{s}, x / c=0.5, \alpha=10^{\circ}, \varphi=0^{\circ}$,
b) $u_{\infty}=30 \mathrm{~m} / \mathrm{s}, x / c=0.8, \alpha=14^{\circ}, \varphi=90^{\circ}$,
c) $u_{\infty}=50 \mathrm{~m} / \mathrm{s}, x / c=0.8, \alpha=14^{\circ}, \varphi=90^{\circ}$ 


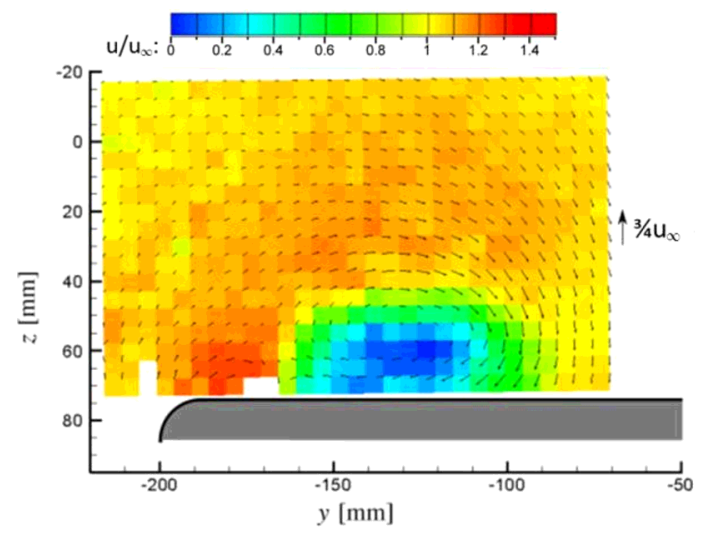

Fig. 11 Actuator model with notched cylinder: velocity field above actuator model $(x / c=0.8)$, enlarged section showing a broken up primary vortex and a second co-rotating vortex near wing tip $\left(u_{\infty}=30 \mathrm{~m} / \mathrm{s}, \alpha=16^{\circ}\right.$, $\varphi=45^{\circ}$.

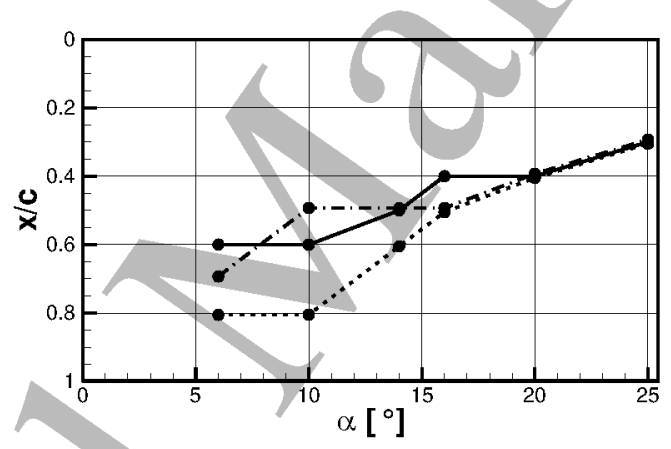

Fig. 12 Actuator model with notched cylinder, position of vortex breakdown $v$ s. angle of attack, $u_{\infty}=30 \mathrm{~m} / \mathrm{s}$ : baseline (solid line), and $\varphi=45^{\circ}$ (dashed line: left wing half, dash-dotted line: right wing half). 
a)

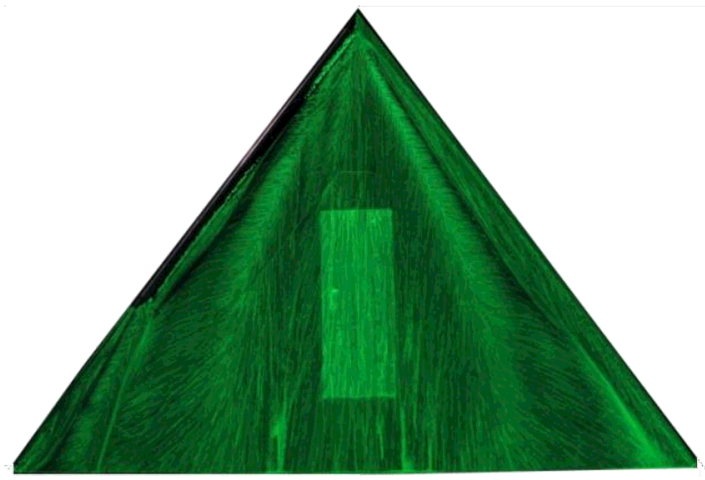

b)

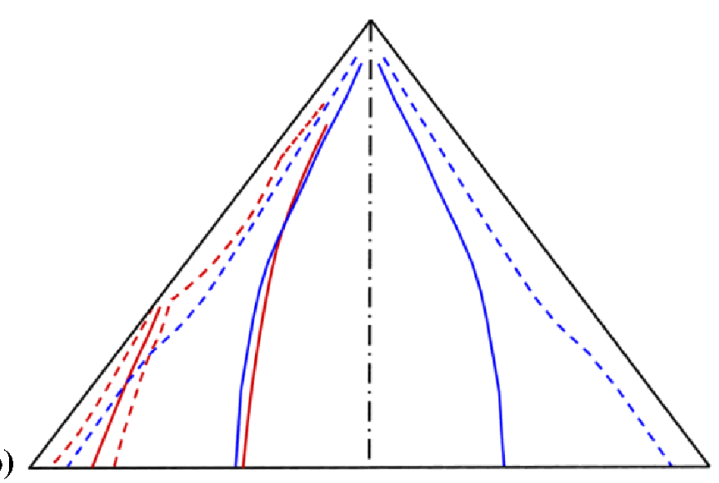

c)
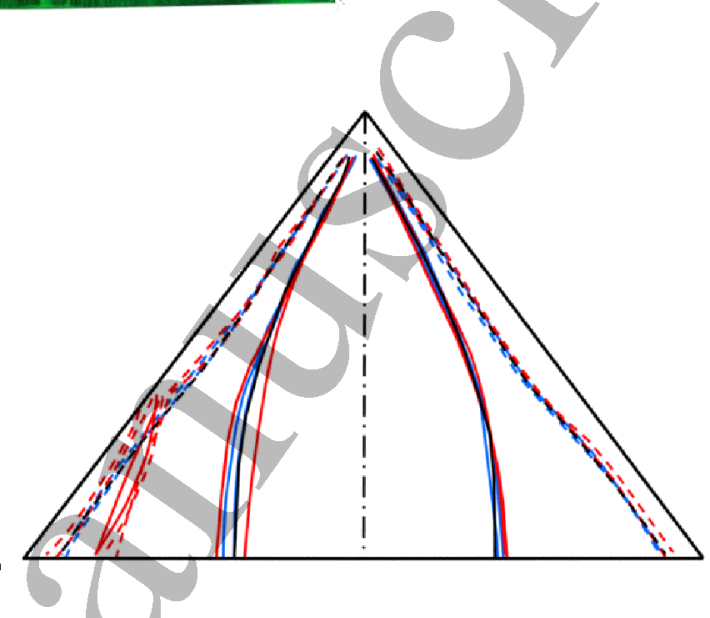

Fig. 13 Actuator model with notched cylinder, skin friction lines on lee side $\left(u_{\infty}=30 \mathrm{~m} / \mathrm{s}, \alpha=16^{\circ}\right)$ :

a) oil-flow image $\left(\varphi=45^{\circ}\right)$,

b) vortex reattachment (solid) and separation lines (dashed), red lines: left wing half, blue lines: right wing half and mirrored to left wing half for comparison $\left(\varphi=45^{\circ}\right)$,

c) vortex reattachment (solid) and separation lines (dashed) for different orientations of notch: $\varphi=180^{\circ}$ (black line), $\varphi=0^{\circ}, 45^{\circ}$ (red lines), $\varphi=-45^{\circ}, 90^{\circ}$ (blue lines). 

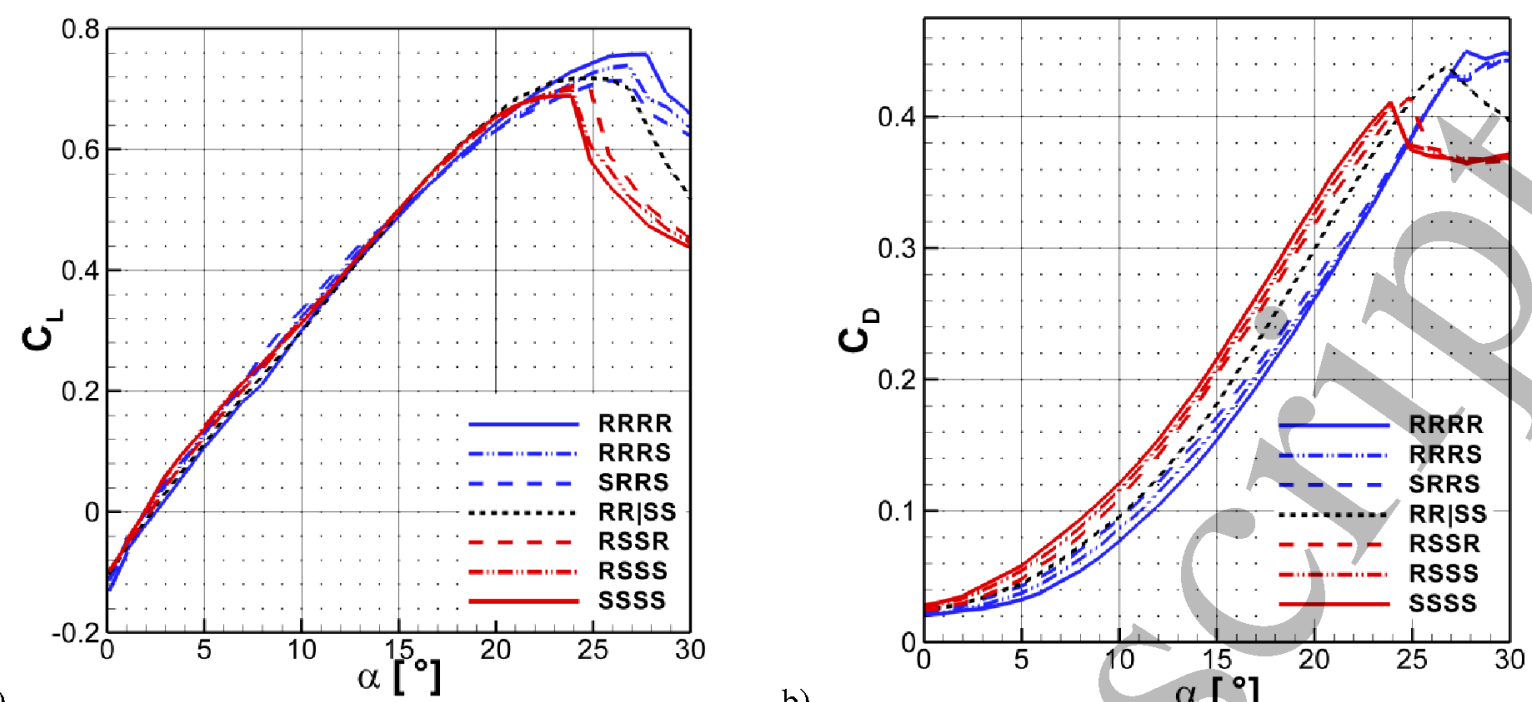

a)

b)

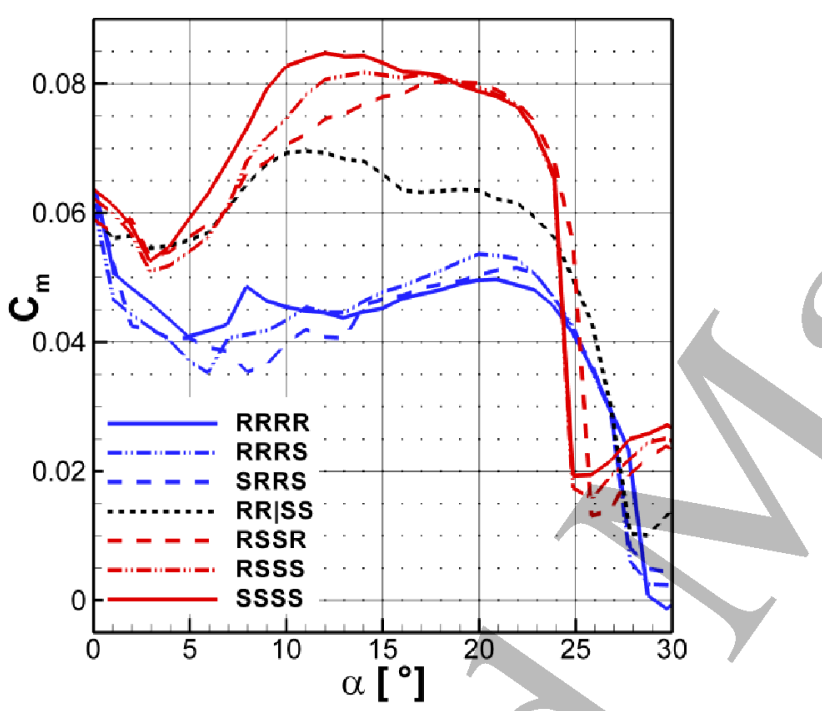

Fig. 14 Thin model with different leading edges: longitudinal coefficients $v s$. AoA $\left(u_{\infty}=30 \mathrm{~m} / \mathrm{s}\right)$.

a) lift coefficient, b) drag coefficient, c) pitching moment coefficient. 
a)

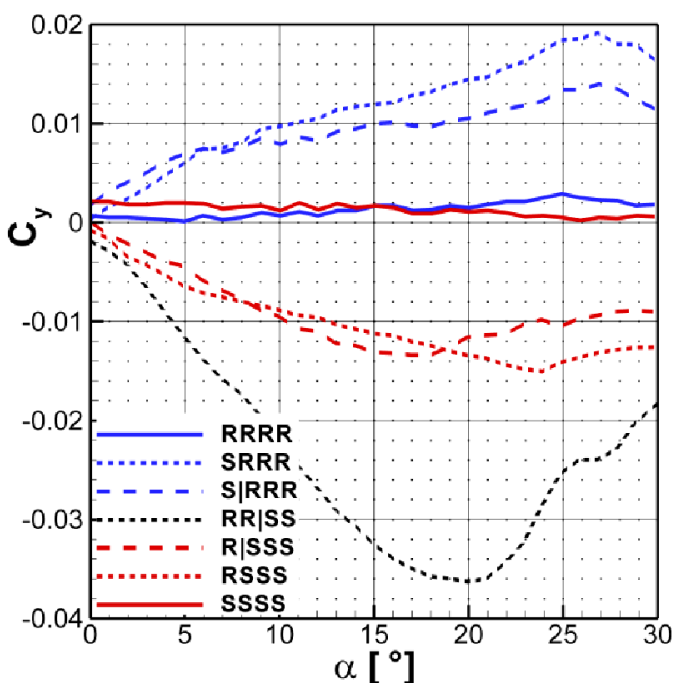

$\alpha\left[^{\circ}\right]$

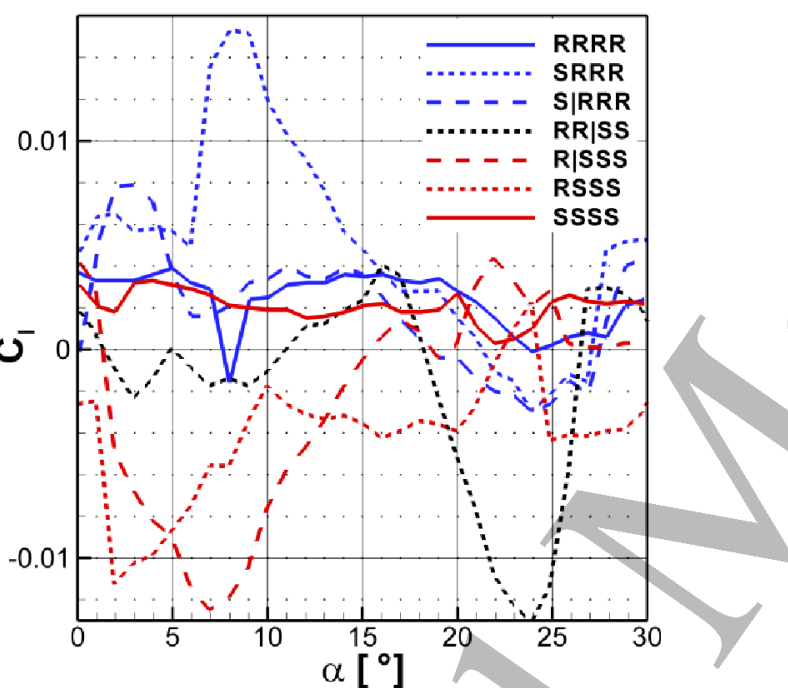

c)

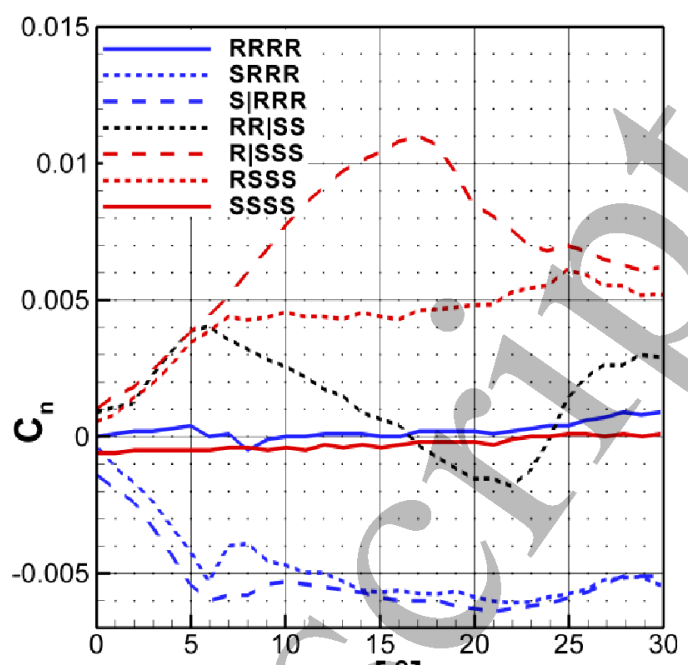

b) $\alpha\left[^{\circ}\right]$

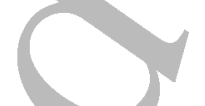

Fig. 15 Thin model with different leading edges: lateral coefficients $v s$. AoA $\left(u_{\infty}=30 \mathrm{~m} / \mathrm{s}\right)$.

a) side force coefficient b) yawing moment coefficient c) rolling moment coefficient. 


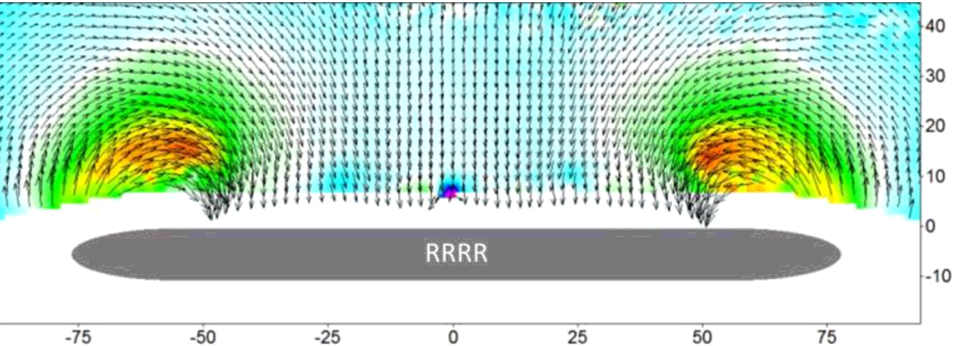

a)

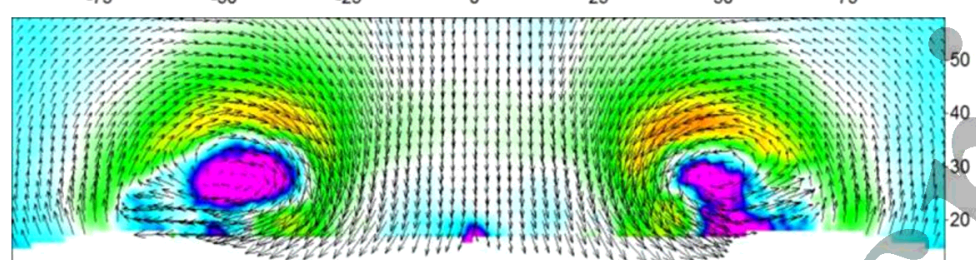

b)

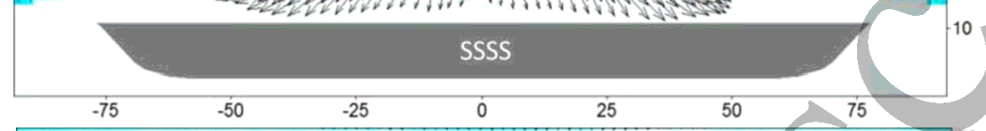

c)
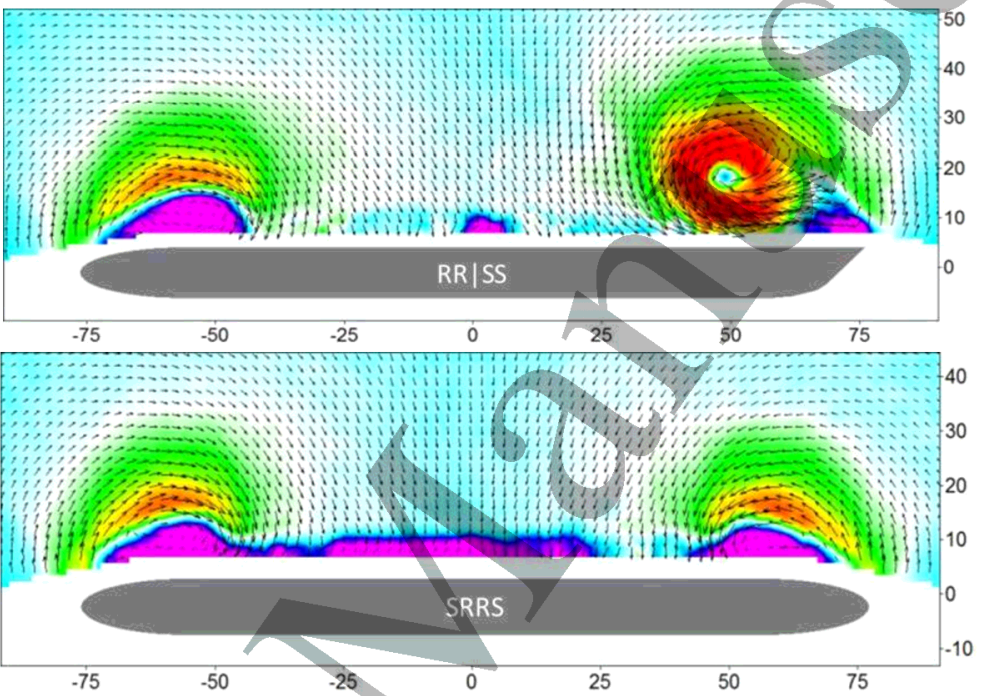

d)

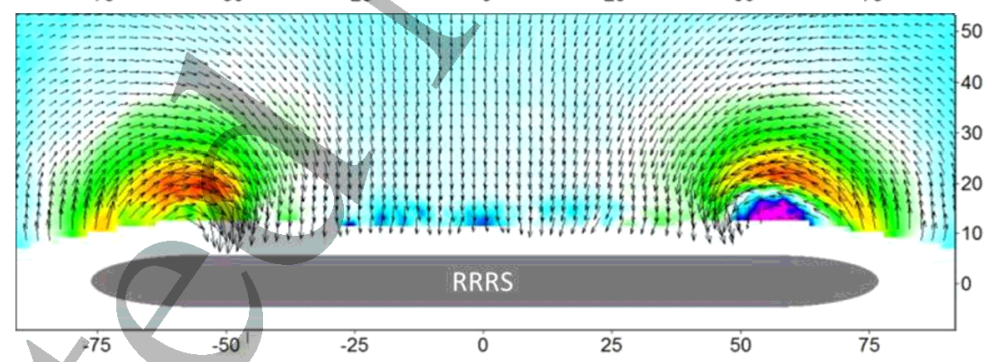

e)
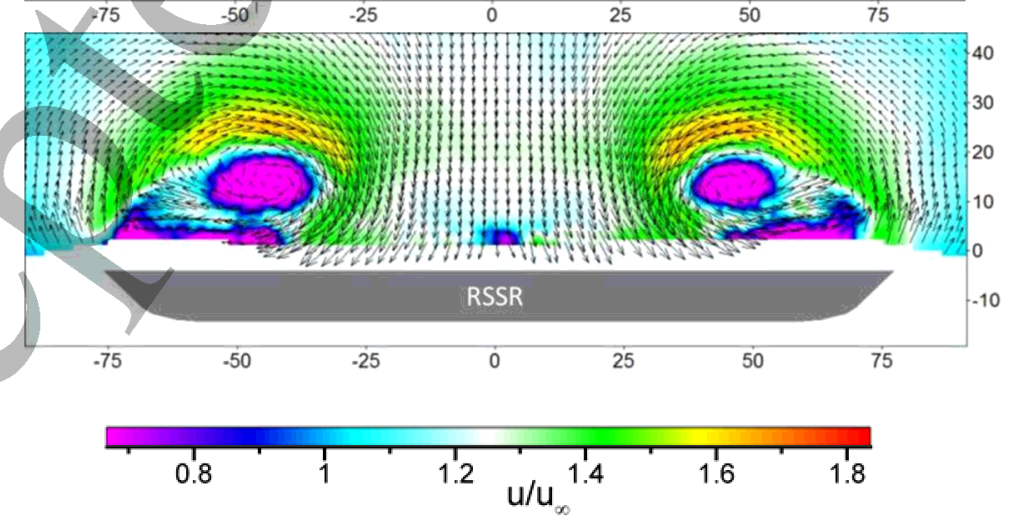

Fig. 16 Thin model with different leading edge configurations: velocity fields above model at $x / c=0.31$ (relative velocity magnitude colour coded, pilot's view), $\mathbf{u}_{\infty}=30 \mathrm{~m} / \mathrm{s}, \alpha=20^{\circ}$. 


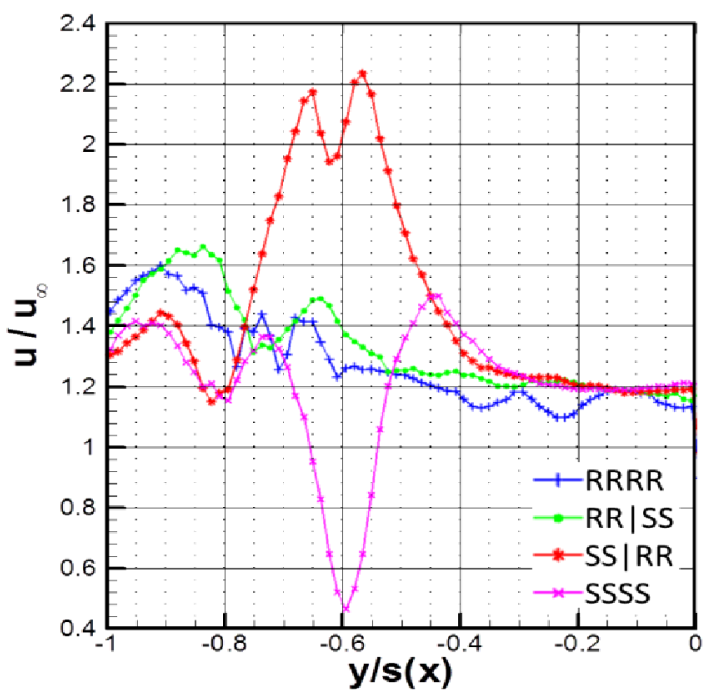

Fig. 17 Thin model with different leading edges: relative velocity magnitude on the lee side of the left wing half $\left(x / c=0.31, u_{\infty}=30 \mathrm{~m} / \mathrm{s}, \alpha=17.5^{\circ}\right)$.

a)

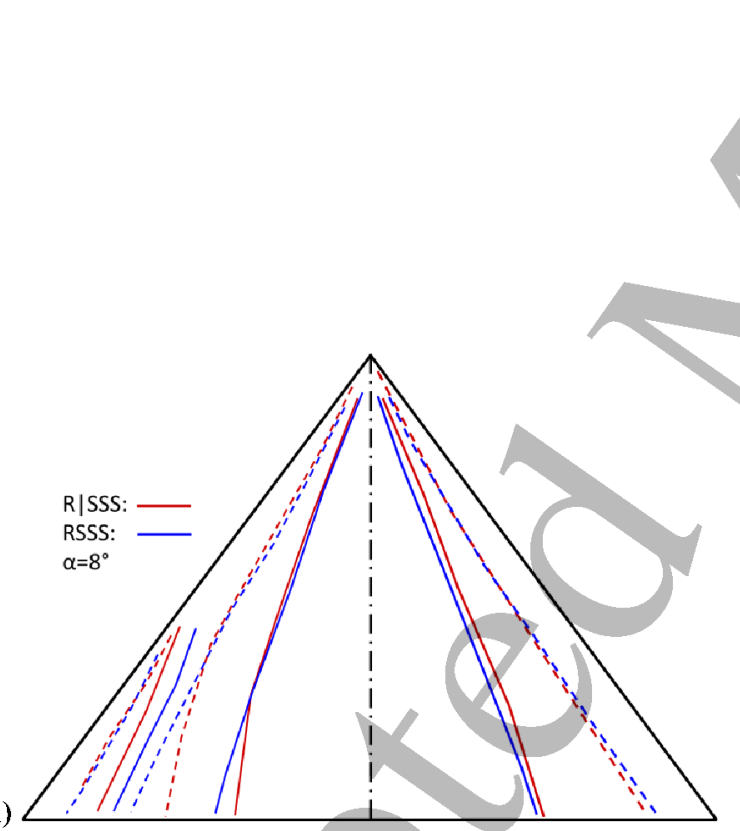

b)

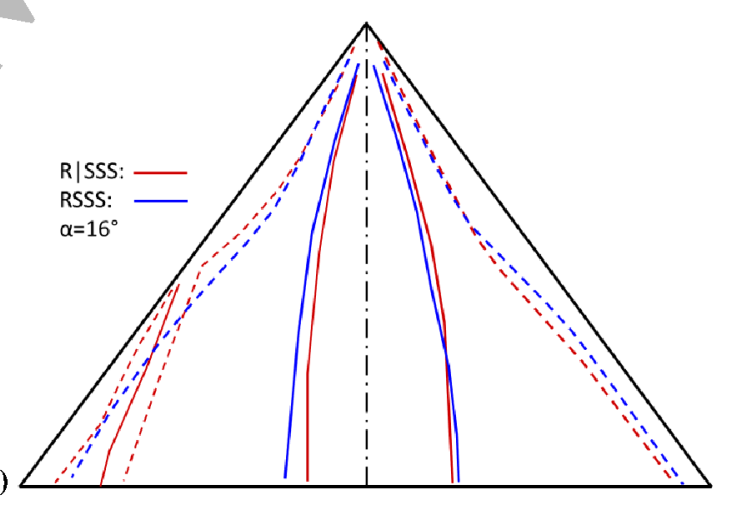

Fig. 18 Thin model, comparison of configurations R/SSS and RSSS $\left(u_{\infty}=30 \mathrm{~m} / \mathbf{s}\right)$ : reattachment (solid lines) and separation (dashed lines); a) $\left.\alpha=8^{\circ}, b\right) \alpha=16^{\circ}$ 
a)

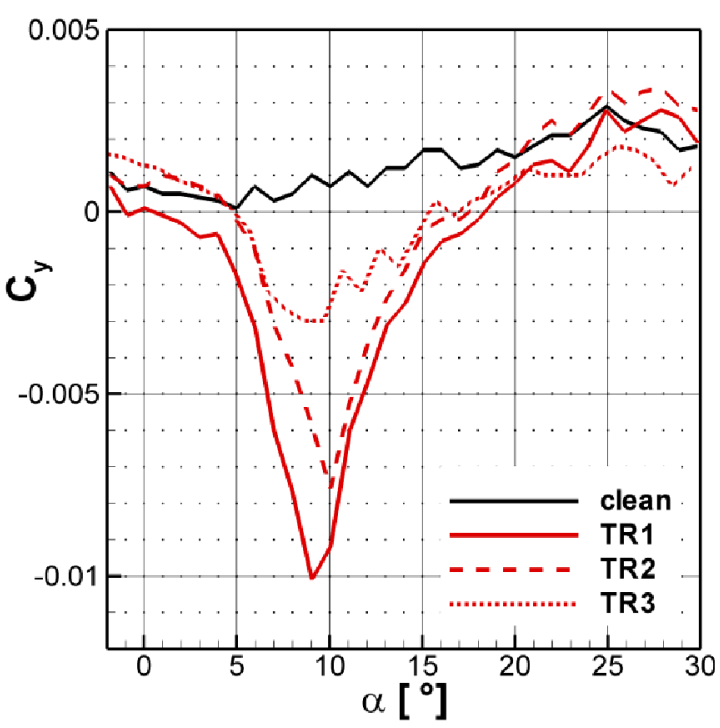

b)

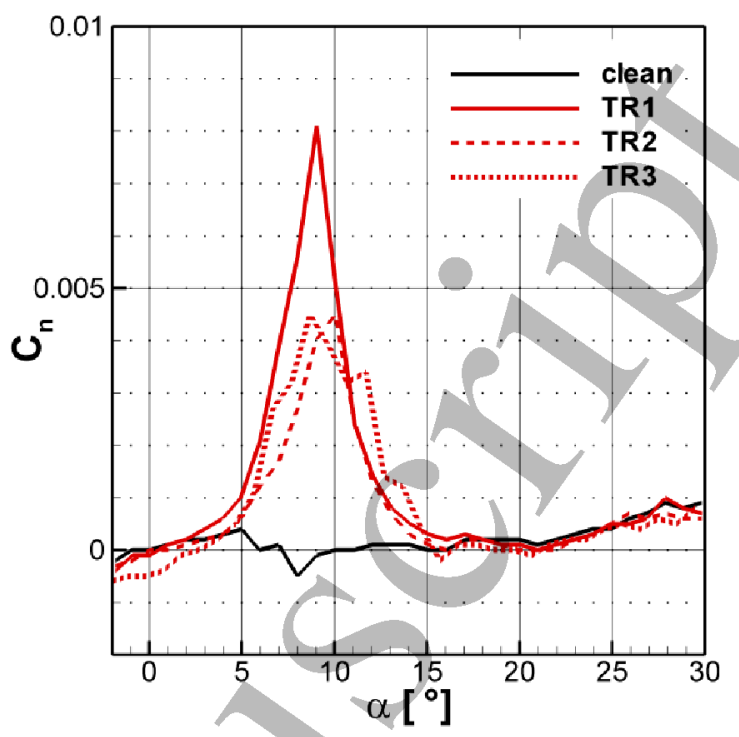

c)

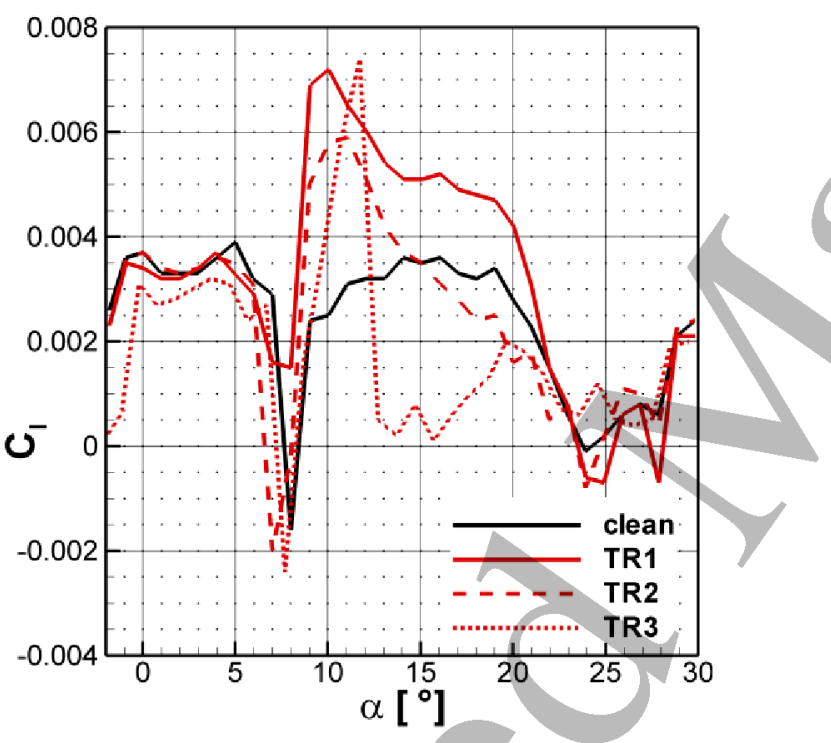

Fig. 19 Thin model (RRRR) with partial tripping on the port side, lateral coefficients $v s$. AoA $\left(u_{\infty}=30 \mathrm{~m} / \mathrm{s}\right)$ : black line: no tripping, solid red line: port side fully tripped (TR1), dashed red line: port side tripped from apex to mid span (TR2), dotted red line: port side tripped from mid span to tip (TR3).

a) side force coefficient, b) yawing moment coefficient, c) rolling moment coefficient. 
a)

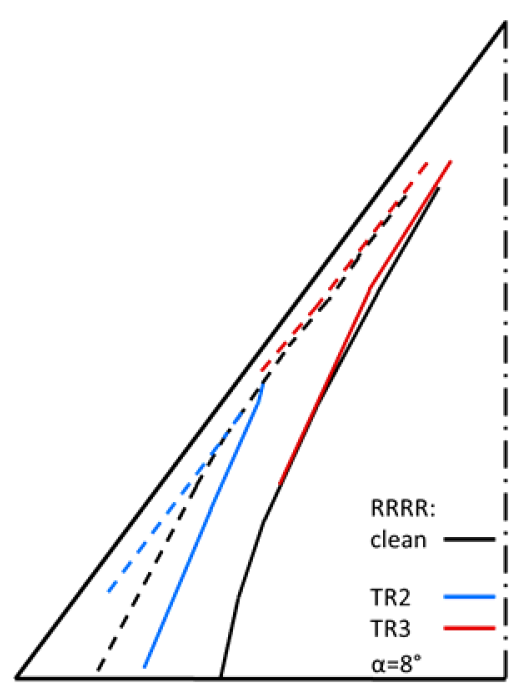

b)

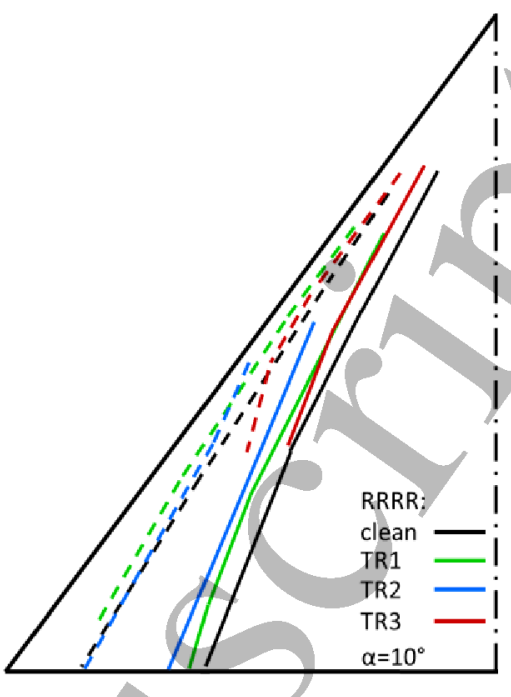

Fig. 20 Thin model: effect of tripping on primary reattachment and secondary separation lines $\left(u_{\infty}=30 \mathrm{~m} / \mathrm{s}\right.$, configuration RRRR), TR1: port side fully tripped, TR2: port side tripped from apex to mid span, TR3: port side tripped from mid span to tip. (a) $\alpha=8^{\circ}$, (b) $\alpha=10^{\circ}$. 